\title{
Precision medicine for mood disorders: objective assessment, risk prediction, pharmacogenomics, and repurposed drugs
}

\author{
H. Le-Niculescu $\mathbb{1}^{1,2} \cdot$ K. Roseberry ${ }^{1} \cdot$ S. S. Gill $\mathbb{1}^{1} \cdot$ D. F. Levey $\mathbb{1}^{1,5} \cdot$ P. L. Phalen $\mathbb{( 1 0}^{1,6} \cdot$ J. Mullen ${ }^{1} \cdot$ A. Williams ${ }^{3} \cdot$

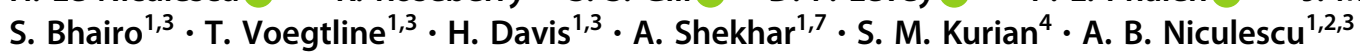

Received: 13 August 2020 / Revised: 8 February 2021 / Accepted: 24 February 2021 / Published online: 8 April 2021

(c) The Author(s) 2021. This article is published with open access

\begin{abstract}
Mood disorders (depression, bipolar disorders) are prevalent and disabling. They are also highly co-morbid with other psychiatric disorders. Currently there are no objective measures, such as blood tests, used in clinical practice, and available treatments do not work in everybody. The development of blood tests, as well as matching of patients with existing and new treatments, in a precise, personalized and preventive fashion, would make a significant difference at an individual and societal level. Early pilot studies by us to discover blood biomarkers for mood state were promising [1], and validated by others [2]. Recent work by us has identified blood gene expression biomarkers that track suicidality, a tragic behavioral outcome of mood disorders, using powerful longitudinal within-subject designs, validated them in suicide completers, and tested them in independent cohorts for ability to assess state (suicidal ideation), and ability to predict trait (future hospitalizations for suicidality) [3-6]. These studies showed good reproducibility with subsequent independent genetic studies [7]. More recently, we have conducted such studies also for pain [8], for stress disorders [9], and for memory/Alzheimer's Disease [10]. We endeavored to use a similar comprehensive approach to identify more definitive biomarkers for mood disorders, that are transdiagnostic, by studying mood in psychiatric disorders patients. First, we used a longitudinal within-subject design and whole-genome gene expression approach to discover biomarkers which track mood state in subjects who had diametric changes in mood state from low to high, from visit to visit, as measured by a simple visual analog scale that we had previously developed (SMS-7). Second, we prioritized these biomarkers using a convergent functional genomics (CFG) approach encompassing in a comprehensive fashion prior published evidence in the field. Third, we validated the biomarkers in an independent cohort of subjects with clinically severe depression (as measured by Hamilton Depression Scale, (HAMD)) and with clinically severe mania (as measured by the Young Mania Rating Scale (YMRS)). Adding the scores from the first three steps into an overall convergent functional evidence (CFE) score, we ended up with 26 top candidate blood gene expression biomarkers that had a CFE score as good as or better than SLC6A4, an empirical finding which we used as a de facto positive control and cutoff. Notably, there was among them an enrichment in genes involved in circadian mechanisms. We further analyzed the biological pathways and networks for the top candidate biomarkers, showing that circadian, neurotrophic, and cell differentiation functions are involved, along with serotonergic and glutamatergic signaling, supporting a view of mood as reflecting energy, activity and growth. Fourth, we tested in independent cohorts of psychiatric patients the ability of each of these 26 top candidate biomarkers to assess state (mood (SMS-7), depression (HAMD), mania (YMRS)), and to predict clinical course (future hospitalizations for depression, future hospitalizations for mania). We conducted our analyses across all patients, as well as personalized by gender and diagnosis, showing increased accuracy with the personalized approach, particularly in women. Again, using SLC6A4 as the cutoff, twelve top biomarkers had the strongest overall evidence for tracking and predicting depression after all four steps: NRG1, DOCK10, GLS, PRPS1, TMEM161B, GLO1, FANCF, HNRNPDL, CD47, OLFM1, SMAD7, and SLC6A4. Of them, six had the strongest overall evidence for tracking and predicting both depression and mania, hence bipolar mood disorders. There were also two biomarkers (RLP3 and SLC6A4) with the strongest overall evidence for mania. These panels of biomarkers have practical implications for distinguishing between depression and bipolar disorder. Next, we evaluated the evidence for our top biomarkers being targets of existing psychiatric drugs, which permits matching patients to medications in a targeted fashion, and the measuring of response to treatment. We also used the biomarker signatures to bioinformatically identify new/repurposed candidate drugs. Top drugs of interest as potential new antidepressants were pindolol, ciprofibrate, pioglitazone and adiphenine, as well as the natural compounds asiaticoside and chlorogenic acid. The last 3 had also been identified by our previous suicidality studies. Finally, we provide an example of how a report to doctors would look for a patient with depression, based on the panel of top biomarkers (12 for depression and bipolar, one for mania), with an objective depression score, risk for future depression, and risk for bipolar switching, as well as personalized lists of targeted prioritized existing psychiatric medications and new potential medications. Overall, our studies provide objective assessments, targeted therapeutics, and monitoring of response to treatment, that enable precision medicine for mood disorders.
\end{abstract}

These authors contributed equally: H. Le-Niculescu, K. Roseberry

Supplementary information The online version contains supplementary material available at https://doi.org/10.1038/s41380021-01061-w.
A. B. Niculescu anicules@iupui.edu

Extended author information available on the last page of the article 


\section{Introduction}

"How weary, stale, flat, and unprofitable

Seem to me all the uses of this world!"

$$
\text { - W. Shakeaspeare, Hamlet }
$$

"There are good and bad times, but our mood changes more often than our fortune."

$$
\text { - Thomas Carlyle }
$$

Mood disorders affect up to 1 in 4 individuals in their lifetime. Depression in particular is the leading cause of disability for ages $15-44$, a prime productive and reproductive age. Due to the lack of objective tests and the perceived presence of stigma, mood disorders are often underdiagnosed or misdiagnosed (depression instead of bipolar disorder). They are also sub-optimally treated, can lead to self-medication with alcohol and drugs, and may culminate in some cases with suicide.

Blood biomarkers are emerging as important tools in disorders where subjective self-report of an individual or clinical impression of a healthcare professional are not always reliable, and for predicting future risk before the disorder (re-)occurs. They also open the door to precise, personalized matching with medications, and objective monitoring of response to treatment. Pioneering early work by our group has identified candidate blood gene expression biomarkers for mood state using a case-case design and a visual analog scale (VAS) (Le-Niculescu et al.) [1]. Those biomarkers were also validated independently as tracking response to cognitive-behavioral therapy by another group [2]. Recent work by our group has identified blood gene expression biomarkers that track suicidality, a tragic outcome of mood disorders, using a new powerful withinsubject longitudinal stepwise approach [4, 5, 11]. These studies show good reproducibility and provide a Rosetta Stone for recent multiple genetic studies of suicide (GWAS, family based) [7]. More recently, we have conducted such studies for pain [8], for stress disorders [9], and for memory/ Alzheimer's Disease [10].

We endeavored to use a similar comprehensive approach to identify more definitive biomarkers for mood disorders in general, and depression in particular. Psychiatric patients may have an increased vulnerability to mood disorders, regardless of their primary diagnosis, as well as increased reasons for mood disorders, due to their often-adverse life trajectory. As such, they may be a particularly suitable population in which to try to identify blood biomarkers for mood disorders, that are generalizable and transdiagnostic. First, we used a powerful longitudinal within-subject design (Fig. 1 and Table 1) in individuals with psychiatric disorders to discover blood gene expression changes between self-reported low mood and high mood states, measured by a VAS, called the Simplified Affective State Scale (SASS), previously described by us $[4,5,11,12]$, which has a subscale of seven items related to mood (SMS-7) (Fig. S1). Second, we prioritized this list of candidate biomarkers with a Bayesian-like CFG approach, comprehensively integrating previous human and animal model evidence in the field. Third, we validated our top candidate biomarkers for mood from discovery and prioritization in an independent cohort of psychiatric subjects with clinically severe depression (as measured by HAMD) or with clinically severe mania (as measured by YMRS). We also analyzed the biological pathways and networks they are involved in (Table 2). Fourth, we tested if the top candidate biomarkers from the first three steps are able to predict low mood state, clinical depression state, and future hospitalizations with depression, in another independent cohort of psychiatric subjects. We tested the biomarkers in all subjects in the test cohort, as well as in a more personalized fashion by gender and psychiatric diagnosis (Fig. 2A-D). We also conducted similar analyses for predictions of high mood, clinical mania state, and future hospitalizations with mania (Table 3B, C, and Supplementary Information-Pathways, Predictions and Reproducibility). Next, we identified which of our biomarkers are targets of existing drugs and thus can be used for pharmacogenomic population stratification and measuring of response to treatment for depression. We also used the biomarker gene expression signatures to interrogate the Connectivity Map database from Broad/MIT, and the NIH LINCS database, in order to identify drugs and natural compounds that can be repurposed for treating and preventing depression, including bipolar depression. Finally, we provide an example of how a personalized patient report can be generated for clinicians to use, reflecting the objective assessment of depression state, future risk of severe depression, risk of bipolarity, matching with existing psychiatric medications, matching with non-psychiatric/repurposed medications, and monitoring response to treatment.

\section{Materials and methods}

\section{Cohorts}

We used three independent cohorts: (1) discovery (a longitudinal psychiatric subjects cohort with diametric changes in mood state from at least two consecutive testing visits); (2) validation (an independent psychiatric subjects cohort with clinically severe depression or mania); and (3) testing (an independent psychiatric subjects test cohort for predicting mood state, clinical depression or mania, and for predicting future hospitalizations for depression or mania) (Fig. 1A and Table 1). 
A.

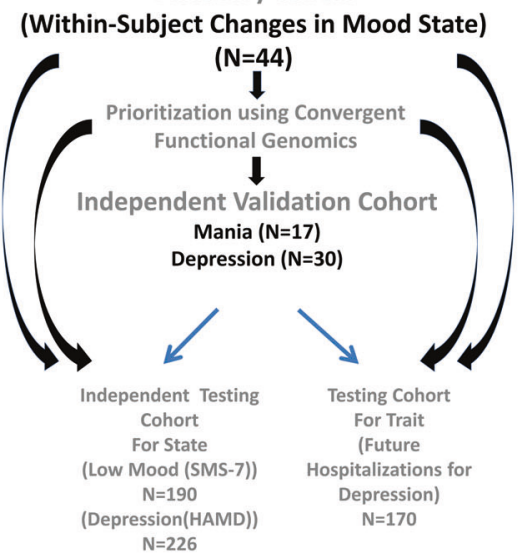

B.

\section{Discovery Cohort:}

30 male and 14 female psychiatric subjects who have at least one switch between a Low Mood state visit and a High Mood state visit.

\section{Male Subjects}

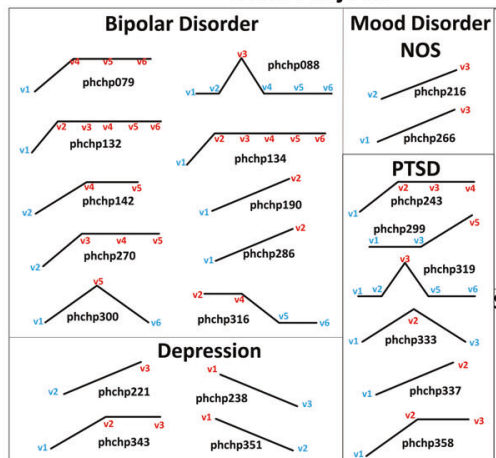

Female Subjects
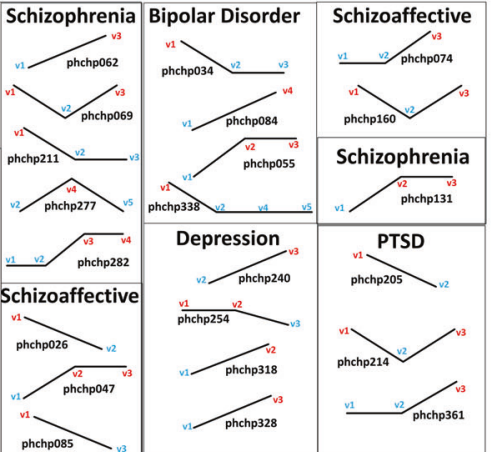

C.

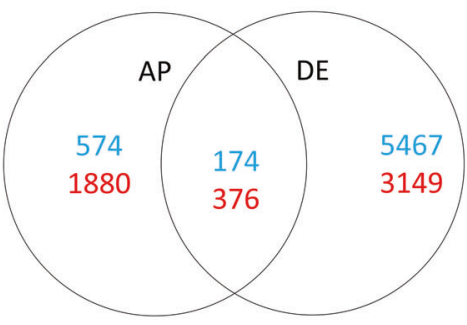

D.

\section{Convergent Functional Genomics}

Multiple Independent Lines of Evidence
For Identification and Prioritization of Mood Bio

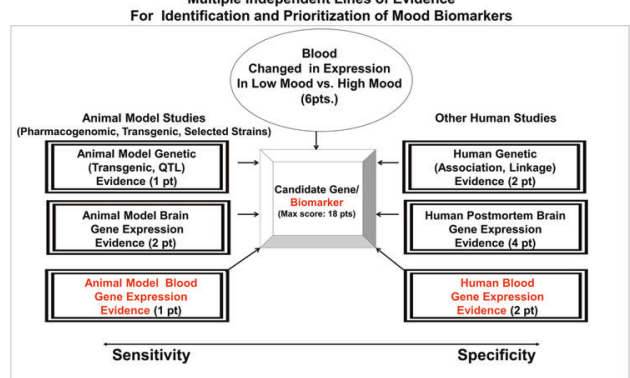

E.

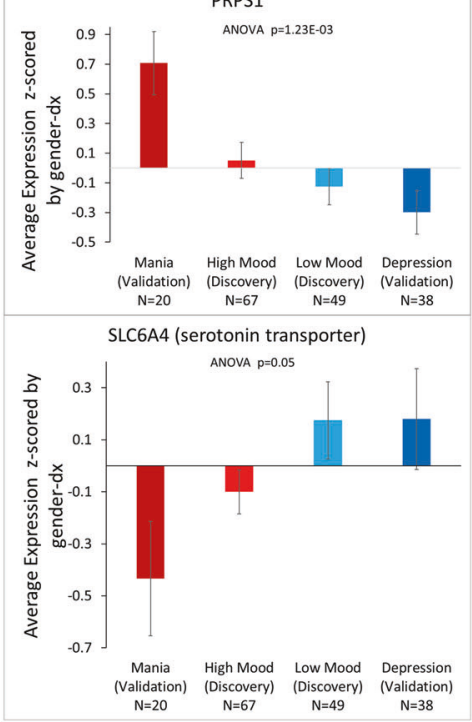

Discovery

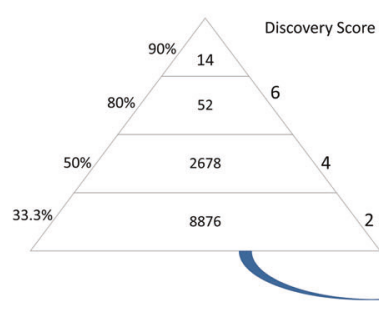

Prioritization

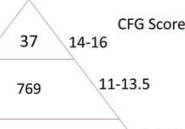

Fig. 1 Steps 1-3: Discovery, Prioritization and Validation of Biomarkers for Mood. A Cohorts used in study, depicting flow of discovery, prioritization, and validation of biomarkers from each step. B Discovery cohort longitudinal within-subject analysis. Phchp\#\#\# is study ID for each subject. V\# denotes visit number. C Differential gene expression in the Discovery cohort- number of genes identified with differential expression (DE) and absent-present (AP) methods with an internal score of 2 and above. Red increased in expression in high mood, blue decreased in expression in high mood. At the discovery step probesets are identified based on their score for tracking mood with a maximum of internal points of $6(33 \%$ (2pt), 50\% (4pt) and 80\% (6pt)). D Prioritization with CFG for prior evidence of involvement in mood disorders. In the prioritization step probesets are converted to their associated genes using Affymetrix annotation and GeneCards. Genes are prioritized and scored using CFG for mood evidence with a maximum of 12 points. Genes scoring at least 6 points out of a maximum possible of 18 total discovery
Validation Convergent Functional Evidence Top CFE Score Validation

\section{.}


Table 1 Demographics of cohorts used.

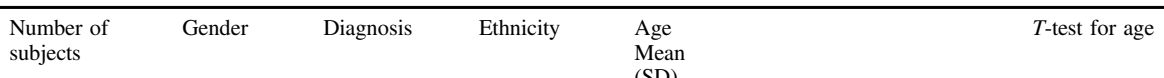

(SD)

\begin{tabular}{|c|c|c|c|c|c|c|}
\hline \multicolumn{7}{|l|}{ Discovery } \\
\hline $\begin{array}{l}\text { Discovery cohort } \\
\text {-within-subject changes in mood } \\
\text { (SMS-7) }\end{array}$ & $\begin{array}{l}44 \\
\text { (with } 134 \text { visits) }\end{array}$ & $\begin{array}{l}\text { Male }=30 \\
\text { Female }=14\end{array}$ & $\begin{array}{l}\mathrm{BP}=14 \\
\mathrm{MDD}=8 \\
\mathrm{SZA}=5 \\
\mathrm{SZ}=6 \\
\mathrm{PTSD}=8 \\
\mathrm{MOOD}=2\end{array}$ & $\begin{array}{l}\mathrm{EA}=33 \\
\mathrm{AA}=9 \\
\text { Asian }=1 \\
\text { Hispanic }=1\end{array}$ & $\mathrm{All}=50.76(6.48)$ & \\
\hline \multicolumn{7}{|l|}{ Validation } \\
\hline $\begin{array}{l}\text { Independent validation cohort } \\
\text {-clinically severe depression } \\
(\mathrm{HAMD} \geq 22)\end{array}$ & 30 & $\begin{array}{l}\text { Male }=23 \\
\text { Female }=7\end{array}$ & $\begin{array}{l}\mathrm{BP}=12 \\
\mathrm{MDD}=12 \\
\mathrm{SZA}=2 \\
\mathrm{PTSD}=3 \\
\mathrm{PSYCH}=1\end{array}$ & $\begin{array}{l}\mathrm{EA}=27 \\
\mathrm{AA}=2 \\
\text { Asian }=1\end{array}$ & $\begin{array}{l}\text { All }=49.42 \\
(7.06)\end{array}$ & \\
\hline $\begin{array}{l}\text { Independent validation cohort } \\
\text {-clinically severe mania } \\
\text { (YMRS } \geq 20 \text { ) }\end{array}$ & 17 & $\begin{array}{l}\text { Male }=16 \\
\text { Female }=1\end{array}$ & $\begin{array}{l}\mathrm{BP}=8 \\
\mathrm{SZA}=6 \\
\mathrm{SZ}=2 \\
\mathrm{PSYCH}=1\end{array}$ & $\begin{array}{l}\mathrm{EA}=11 \\
\mathrm{AA}=6\end{array}$ & $\begin{array}{l}\text { All }=48.25 \\
(8.21)\end{array}$ & \\
\hline \multicolumn{7}{|l|}{ Testing } \\
\hline $\begin{array}{l}\text { Independent testing cohort } \\
\text { State predictions } \\
\text { Low Mood } \\
(\text { SMS-7 } \leq 40)\end{array}$ & 190 & $\begin{array}{l}\text { Male }=153 \\
\text { Female }=37\end{array}$ & $\begin{array}{l}\mathrm{BP}=52 \\
\mathrm{MDD}=30 \\
\mathrm{SZA}=48 \\
\mathrm{SZ}=36 \\
\mathrm{PTSD}=16 \\
\mathrm{MOOD}=5 \\
\mathrm{PSYCH}=3\end{array}$ & $\begin{array}{l}\mathrm{EA}=118 \\
\mathrm{AA}=69 \\
\text { Hispanic }=2 \\
\text { Mixed }=1\end{array}$ & $\begin{array}{l}\text { All }=50.52 \\
(8.58) \\
\text { Low } \operatorname{mood}=49(n=87) \\
\text { Others }=50.88(n=359\end{array}$ & $\begin{array}{l}T \text {-test for age between low mood vs. } \\
\text { Others } 0.10469\end{array}$ \\
\hline $\begin{array}{l}\text { Independent testing cohort } \\
\text { State predictions } \\
\text { Clinical Depression }(H A M D \geq 22 \text { ) }\end{array}$ & 226 & $\begin{array}{l}\text { Male }=181 \\
\text { Female }=45\end{array}$ & $\begin{array}{l}\mathrm{BP}=74 \\
\mathrm{MDD}=39 \\
\mathrm{SZA}=48 \\
\mathrm{SZ}=36 \\
\mathrm{PTSD}=21 \\
\mathrm{MOOD}=5 \\
\mathrm{PSYCH}=3\end{array}$ & $\begin{array}{l}\mathrm{EA}=156 \\
\mathrm{AA}=66 \\
\text { Asian }=1 \\
\text { Hispanic= } 2 \\
\text { Mixed =1 }\end{array}$ & $\begin{array}{l}\text { All }=46.71 \\
(9.42) \\
\text { Clinical depression }=44.4(n=40) \\
\text { Others }=46.9(n=445)\end{array}$ & $\begin{array}{l}T \text {-test for age between clinical } \\
\text { depression vs. others } 0.177087984\end{array}$ \\
\hline $\begin{array}{l}\text { Independent testing cohort } \\
\text { Trait predictions } \\
\text { Hospitalizations with Depression } \\
\text { First year following initial visit }\end{array}$ & 147 & $\begin{array}{l}\text { Male }=130 \\
\text { Female }=17\end{array}$ & $\begin{array}{l}\mathrm{BP}=37 \\
\mathrm{MDD}=27 \\
\mathrm{SZA}=32 \\
\mathrm{SZ}=33 \\
\mathrm{PTSD}=13 \\
\mathrm{MOOD}=3 \\
\mathrm{PSYCH}=2\end{array}$ & $\begin{array}{l}\mathrm{EA}=90 \\
\mathrm{AA}=54 \\
\text { Mixed }=1 \\
\text { Hispanic }=2\end{array}$ & $\begin{array}{l}\text { All }=47.13 \\
(9.38) \\
\text { Hosp with Depression }=46.58(n=50) \\
\text { Others }=47.23(n=282)\end{array}$ & $\begin{array}{l}T \text {-test for age between hosp with } \\
\text { depression vs. others } \\
0.701909278\end{array}$ \\
\hline $\begin{array}{l}\text { Independent testing cohort } \\
\text { Trait predictions } \\
\text { Hospitalizations with Depression } \\
\text { All future years following initial visit }\end{array}$ & 170 & $\begin{array}{l}\text { Male }=150 \\
\text { Female }=20\end{array}$ & $\begin{array}{l}\mathrm{BP}=41 \\
\mathrm{MDD}=29 \\
\mathrm{SZA}=40 \\
\mathrm{SZ}=39 \\
\mathrm{PTSD}=14 \\
\mathrm{MOOD}=5 \\
\mathrm{PSYCH}=2\end{array}$ & $\begin{array}{l}\mathrm{EA}=102 \\
\mathrm{AA}=65 \\
\text { Mixed }=1 \\
\text { Hispanic }=2\end{array}$ & $\begin{array}{l}\text { All }=49.4 \\
(9.78) \\
\text { Hosp with depression }=49.3(n=127) \\
\text { Others }=49.4(n=282)\end{array}$ & $\begin{array}{l}T \text {-test for age between } \\
\text { hosp with depression vs. others } \\
0.93467396\end{array}$ \\
\hline $\begin{array}{l}\text { Independent testing cohort } \\
\text { State predictions High Mood } \\
(\text { SMS-7 } \geq 60 \text { ) }\end{array}$ & 190 & $\begin{array}{l}\text { Male }=153 \\
\text { Female }=37\end{array}$ & $\begin{array}{l}\mathrm{BP}=52 \\
\mathrm{MDD}=30 \\
\mathrm{SZA}=48 \\
\mathrm{SZ}=36 \\
\mathrm{PTSD}=16 \\
\mathrm{MOOD}=5 \\
\mathrm{PSYCH}=3\end{array}$ & $\begin{array}{l}\mathrm{EA}=118 \\
\mathrm{AA}=69 \\
\text { Hispanic }=2 \\
\text { Mixed }=1\end{array}$ & $\begin{array}{l}\text { All }=50.52 \\
(8.58) \\
\text { High } \operatorname{mood}=50.6(n=185) \\
\text { Others }=50.5(n=261)\end{array}$ & $\begin{array}{l}T \text {-test for age between high mood } \\
\text { vs. others } \\
0.877948\end{array}$ \\
\hline $\begin{array}{l}\text { Independent testing cohort } \\
\text { State predictions } \\
\text { Clinical Mania } \\
\text { (YMRS } \geq 20 \text { ) }\end{array}$ & 97 & $\begin{array}{l}\text { Male }=73 \\
\text { Female }=24\end{array}$ & $\begin{array}{l}\mathrm{BP}=37 \\
\mathrm{MDD}=13 \\
\mathrm{SZA}=18 \\
\mathrm{SZ}=18 \\
\mathrm{PTSD}=10 \\
\mathrm{MOOD}=1\end{array}$ & $\begin{array}{l}\mathrm{EA}=72 \\
\mathrm{AA}=22 \\
\text { Hispanic }=2 \\
\text { Mixed }=1\end{array}$ & $\begin{array}{l}\text { All }=39.4 \\
(8.83) \\
\text { Clinical Mania }=38.9(n=13) \\
=38.9(n=13) \\
\text { Others }=39.4(n=197)\end{array}$ & $\begin{array}{l}T \text {-test for age between mania } \\
\text { vs. others } \\
0.883113775\end{array}$ \\
\hline $\begin{array}{l}\text { Independent testing cohort } \\
\text { Trait predictions } \\
\text { Hospitalizations with Mania } \\
\text { First year following initial visit }\end{array}$ & 147 & $\begin{array}{l}\text { Male }=130 \\
\text { Female }=17\end{array}$ & $\begin{array}{l}\mathrm{BP}=37 \\
\mathrm{MDD}=27 \\
\mathrm{SZA}=32 \\
\mathrm{SZ}=33 \\
\mathrm{PTSD}=13 \\
\mathrm{MOOD}=3 \\
\mathrm{PSYCH}=2\end{array}$ & $\begin{array}{l}\mathrm{EA}=90 \\
\mathrm{AA}=54 \\
\text { Mixed }=1 \\
\text { Hispanic }=2\end{array}$ & $\begin{array}{l}\text { All }=47.13 \\
(9.38) \\
\text { Hosp with Mania } \\
=45.5(n=11) \\
\text { Other }==47.2(n=321) \mathrm{s} \\
=47.2(n=321)\end{array}$ & $\begin{array}{l}T \text {-test for age between hosp } \\
\text { with mania } \\
\text { vs. others } \\
0.588179\end{array}$ \\
\hline $\begin{array}{l}\text { Independent testing cohort } \\
\text { Trait predictions } \\
\text { Hospitalizations with Mania } \\
\text { All future years following initial visit }\end{array}$ & 117 & $\begin{array}{l}\text { Male }=102 \\
\text { Female }=15\end{array}$ & $\begin{array}{l}\mathrm{BP}=34 \\
\mathrm{MDD}=17 \\
\mathrm{SZA}=26 \\
\mathrm{SZ}=26 \\
\mathrm{PTSD}=11 \\
\mathrm{MOOD}=2 \\
\mathrm{PSYCH}=1\end{array}$ & $\begin{array}{l}\mathrm{EA}=74 \\
\mathrm{AA}=40 \\
\text { Mixed }=1 \\
\text { Hispanic }=2\end{array}$ & $\begin{array}{l}\text { All }=44.39 \\
(9.01) \\
\text { Hosp with Mania } \\
=43.7(n=37) \\
\text { Others } \\
=44.5(n=220)\end{array}$ & $\begin{array}{l}T \text {-test for age between hosp with } \\
\text { mania vs. others } \\
0.692290398\end{array}$ \\
\hline
\end{tabular}

$B P$ bipolar, $M D D$ major depressive disorder, $S Z A$ schizoaffective disorder, $S Z$ schizophrenia, $P T S D$ post-traumatic stress disorder, $M O O D$ mood disorder nos, $P S Y C H$ psychosis nos. 


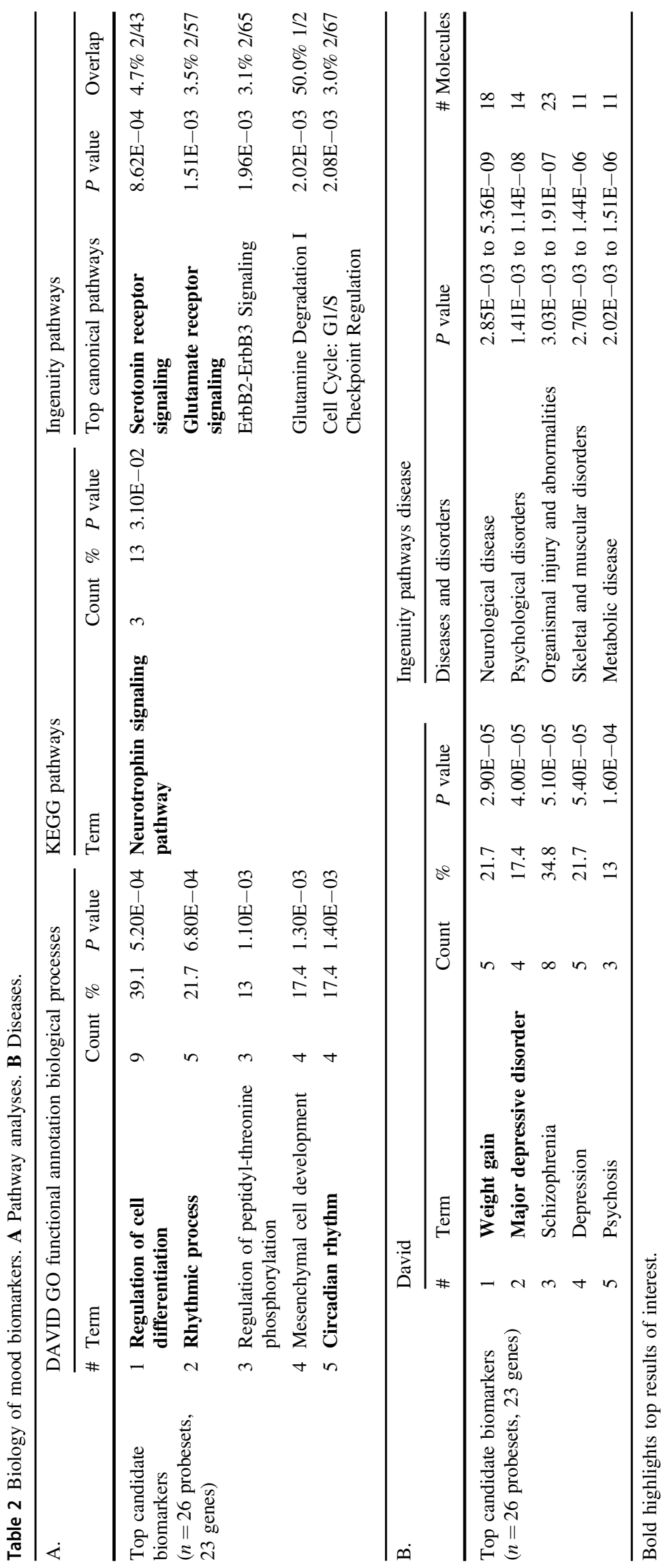


A. Low Mood State Predictions (SMS-7 $\leq 40$ )

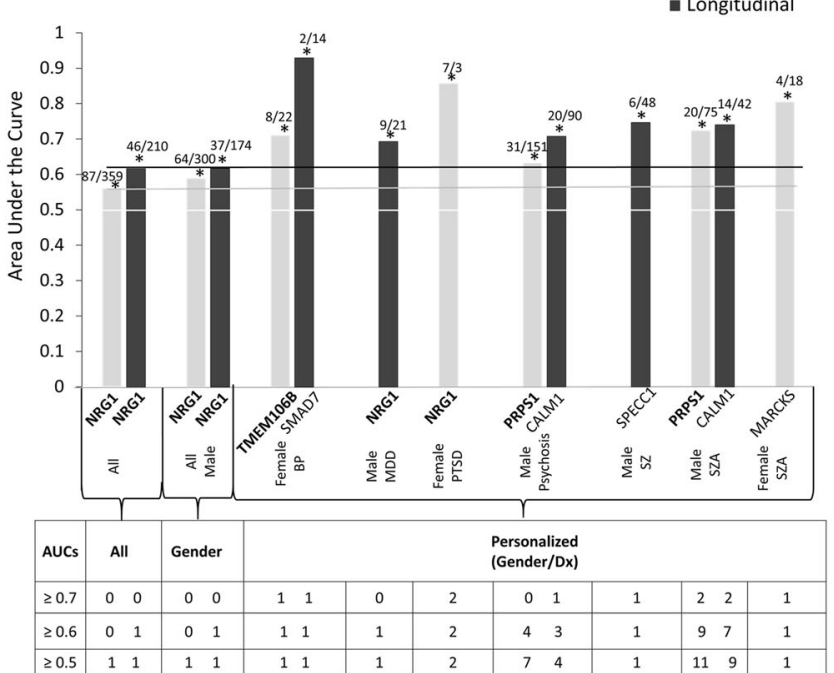

C. Depression Trait Predictions First Year

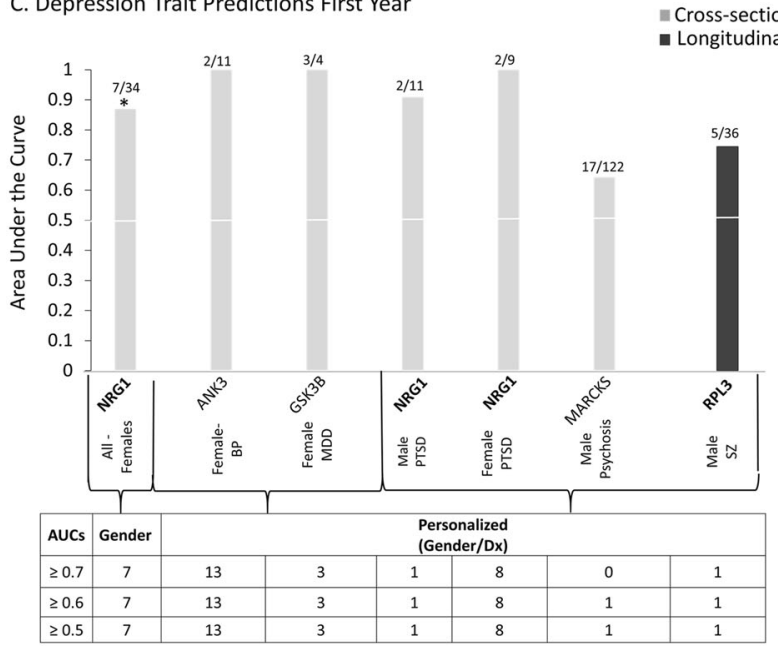

Fig. 2 Best single biomarkers predictors for depression, state and trait. From top candidate biomarkers after Steps 1-3 (discovery, prioritization, validation-bold) $(n=26)$. Bar graph shows best predictive biomarkers in each group. All markers are nominally significant $p<0.05$. Table underneath the figures displays the actual number of biomarkers for each group whose ROC AUC $p$ values $(\mathbf{A}-\mathbf{C}$,) and Cox odds ratio (OR) $p$ values (D) are at least nominally significant. Some gender and diagnosis group are missing from the graph as they did not have any significant biomarkers, or sufficient timepoints in the case of longitudinal predictions. Cross-sectional is

Similar to our previous studies in suicide [3-5], the live psychiatric subjects are part of a larger longitudinal cohort of adults that we are continuously collecting. Subjects are recruited primarily from the patient population at the Indianapolis VA Medical Center. All subjects understood and signed informed consent forms detailing the research goals, procedure, caveats and safeguards, per IRB approved protocol. Subjects completed diagnostic assessments by structured clinical interviews. They had an initial testing visit in the lab or on the inpatient psychiatric unit, followed

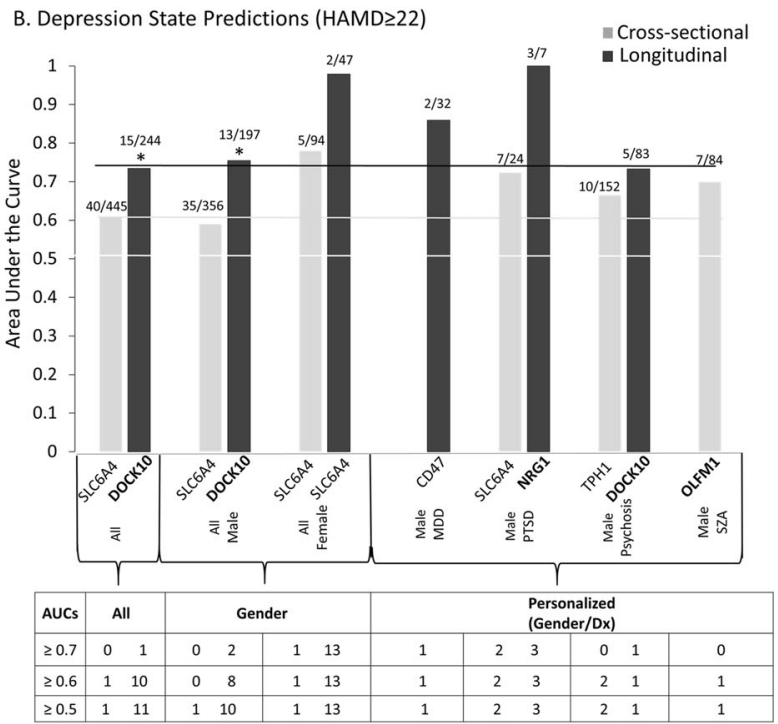

D. Depression Trait Predictions All Future Years

- Cross-sectional

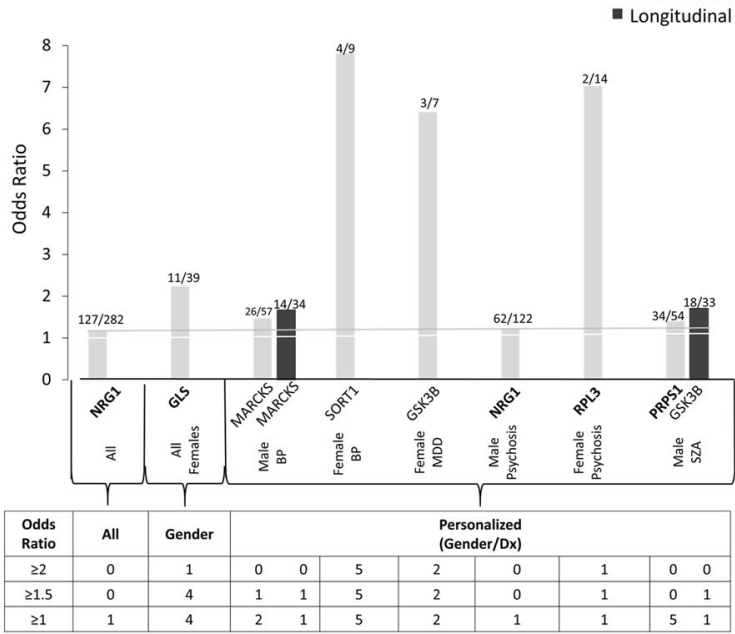

based on levels at one visit. Longitudinal is based on levels at multiple visits (integrates levels at most recent visit, maximum levels, slope into most recent visit, and maximum slope). Dividing lines represent the cutoffs for a test performing at chance levels (white), and at the same level as the best biomarkers for all subjects in cross-sectional (gray) and longitudinal (black) based predictions. All biomarkers perform better than chance. Biomarkers performed better when personalized by gender and diagnosis, particularly in females. $*$ survived Bonferroni correction for the number of candidate biomarkers tested $(n=26)$.

by up to six testing visits, 3-6 months apart or whenever a new psychiatric hospitalization occurred. At each testing visit, they received a series of psychiatric rating scales, and their blood was drawn. The rating scales included the Hamilton Rating Scale for Depression-17 (HAMD), the Young Mania Rating Scale (YMRS), and a visual analog scale for assessing mood state (SMS-7), which provides a score that is the average of seven items (Fig. S1A), and is part of the SASS (Niculescu et al. [12], Niculescu et al. [4], Levey et al. [5], Niculescu et al. [6]). SMS-7 integrates on a 

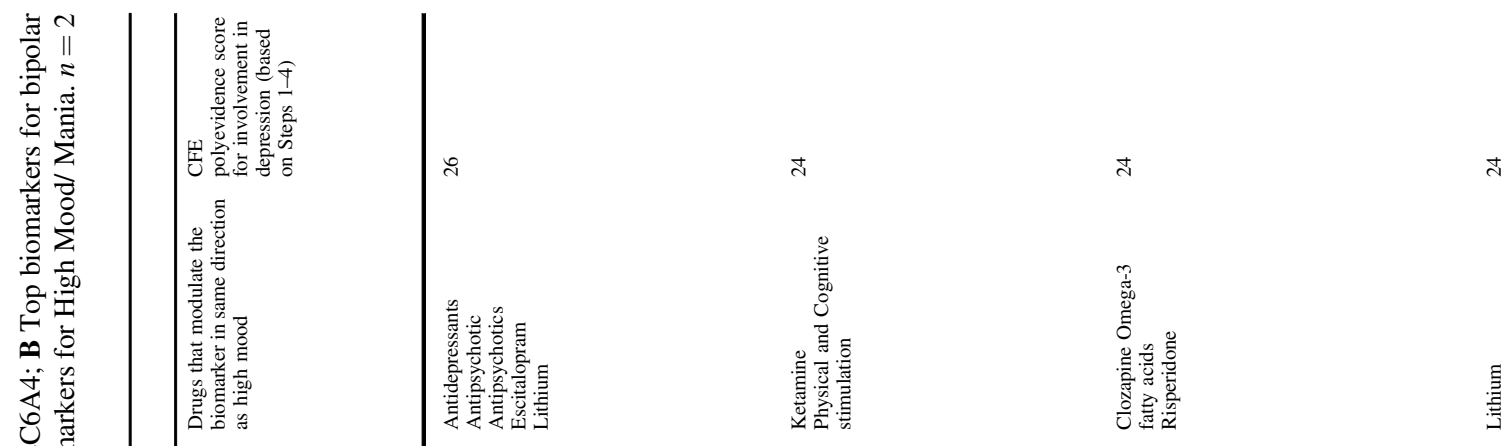

च

?

$\because \quad \frac{8}{5}$

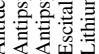

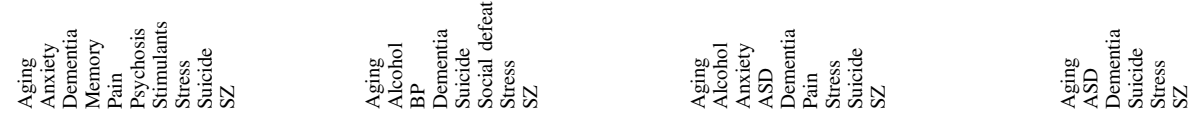

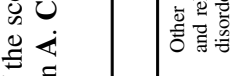

突.

ठ .

สิ एँ

.

si

造

范

$\rightarrow$ 응

过

잉

$\| \stackrel{\Xi}{=}$

들

호욜

ญैँ

웜

उे \&

号

을

t.

过守

䜦㟔

อั อิ

융

$\varangle$

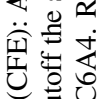

己

कृ

व.

플

过

焉

䒕苍苇

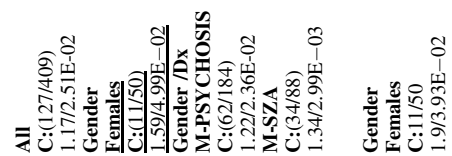

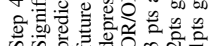

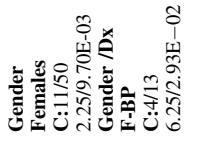

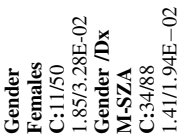

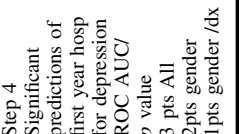

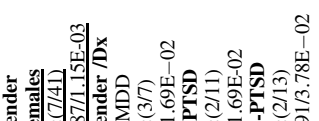

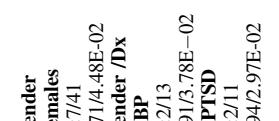

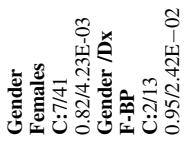

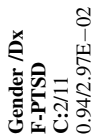

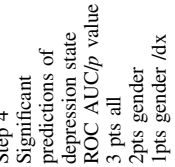

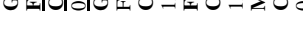

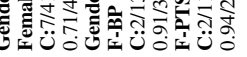

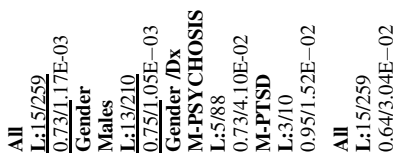

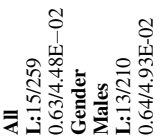

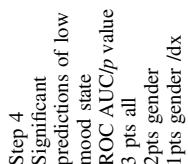

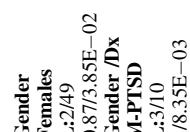

产

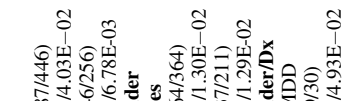

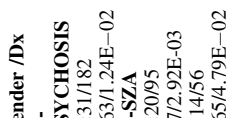

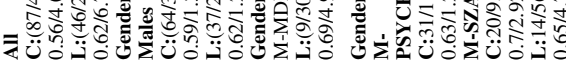

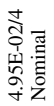

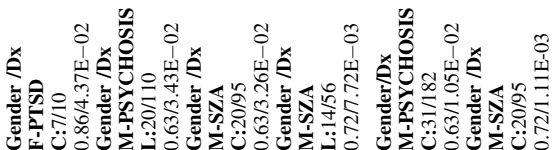

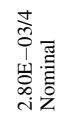

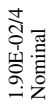

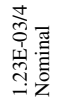

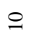

ย啇望

e挡的

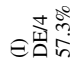

जे
ते
ते

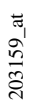

㠃
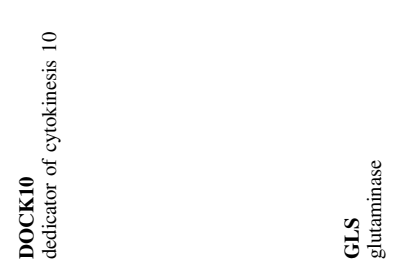

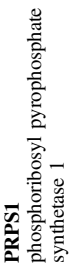




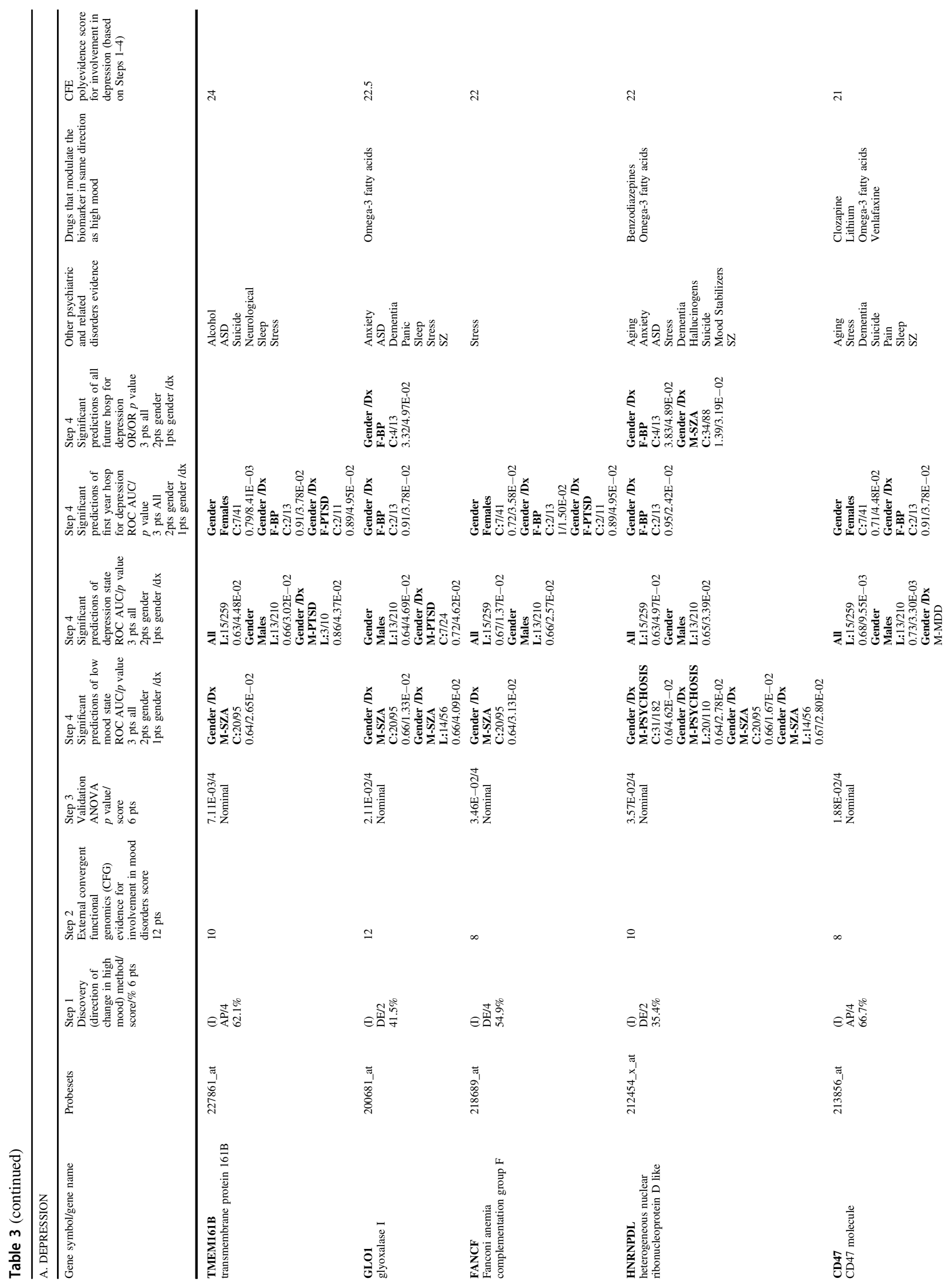




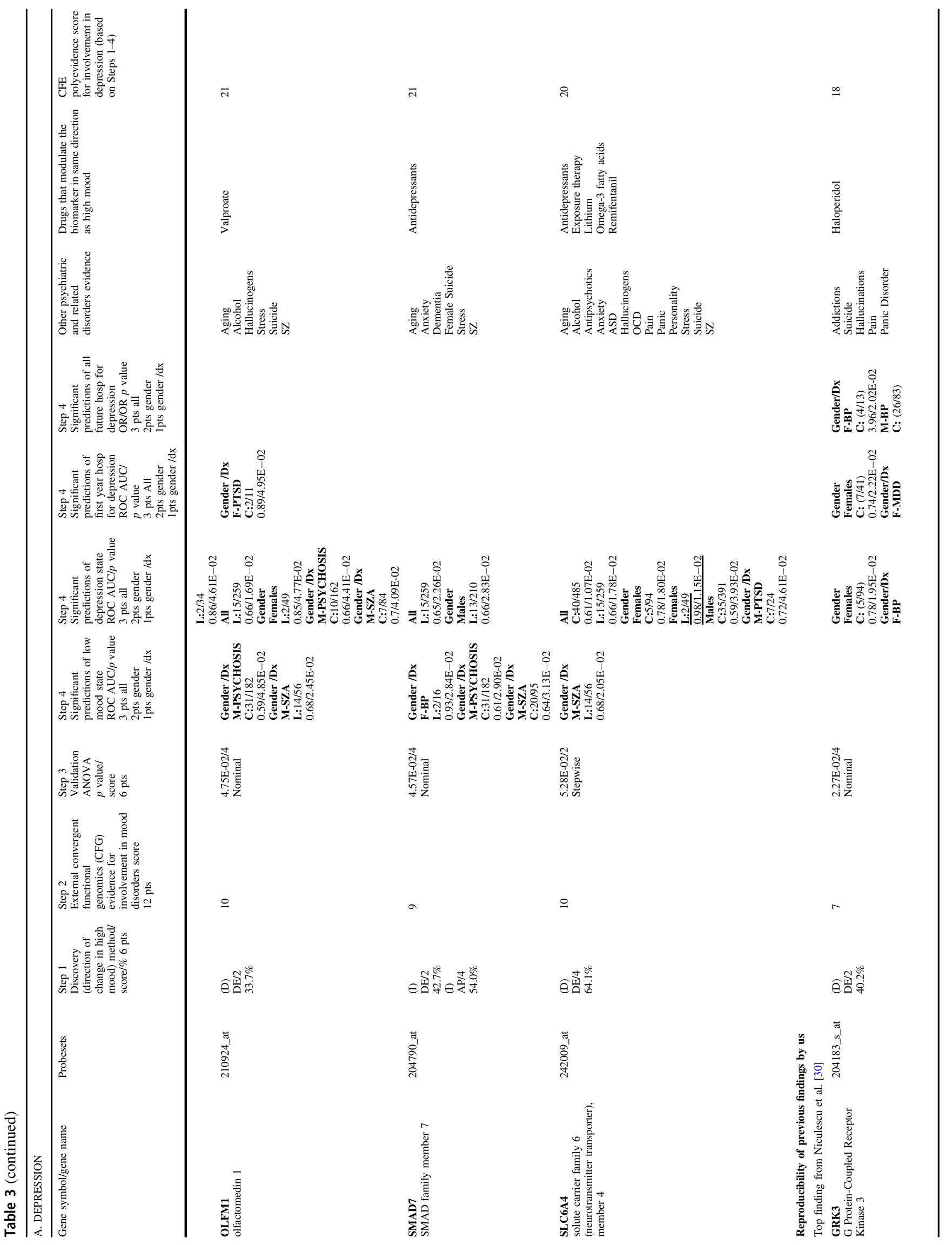




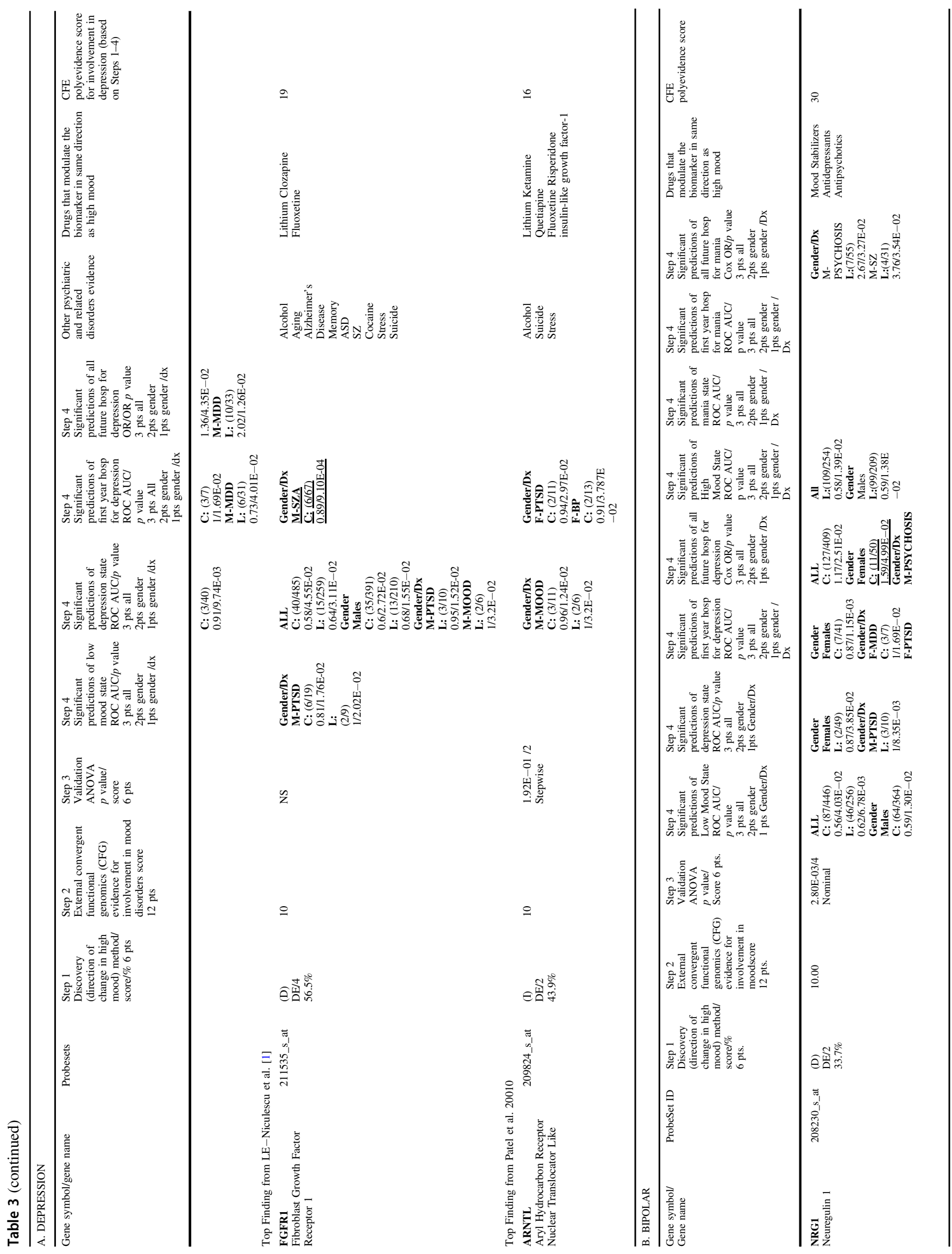




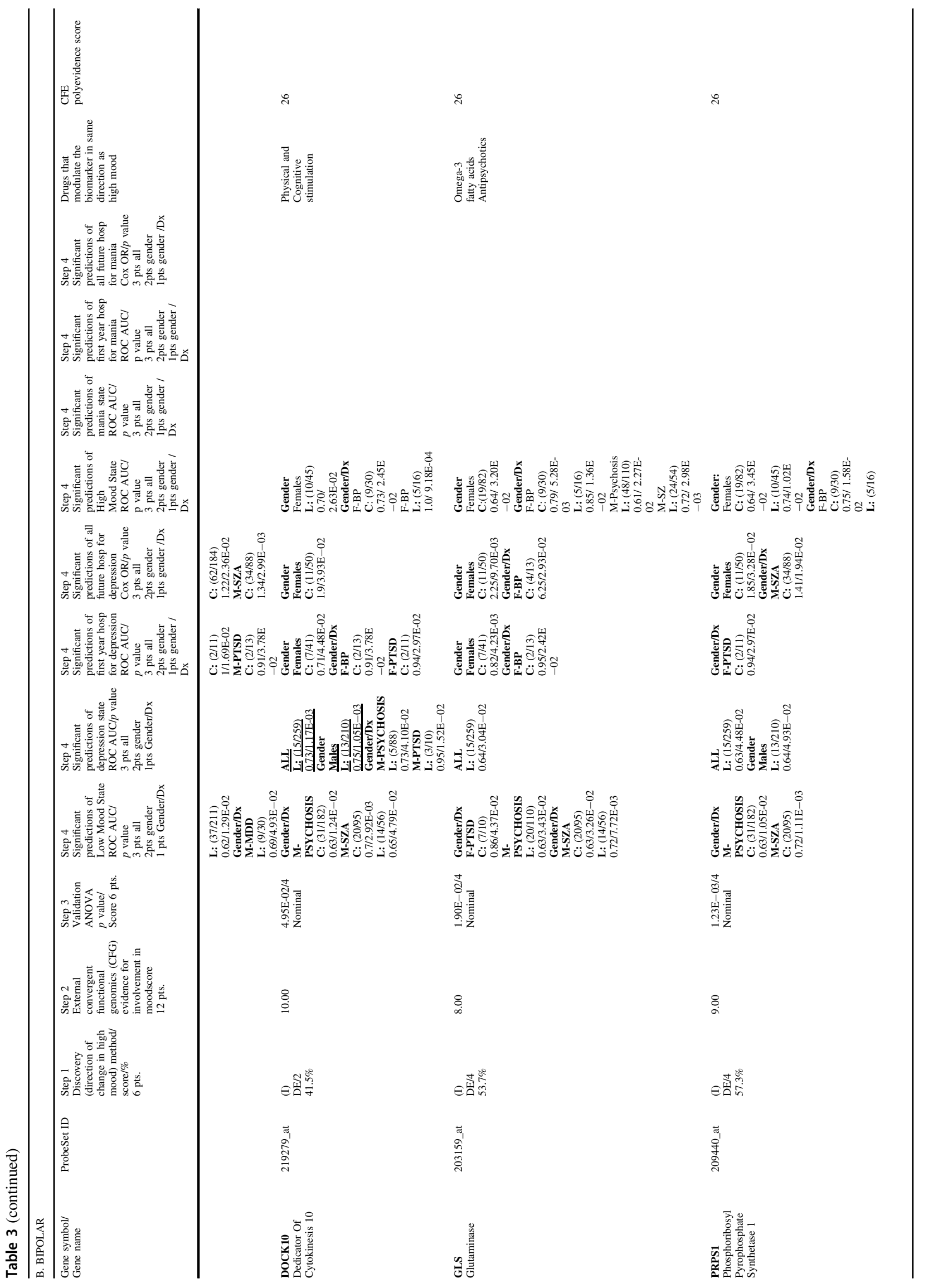




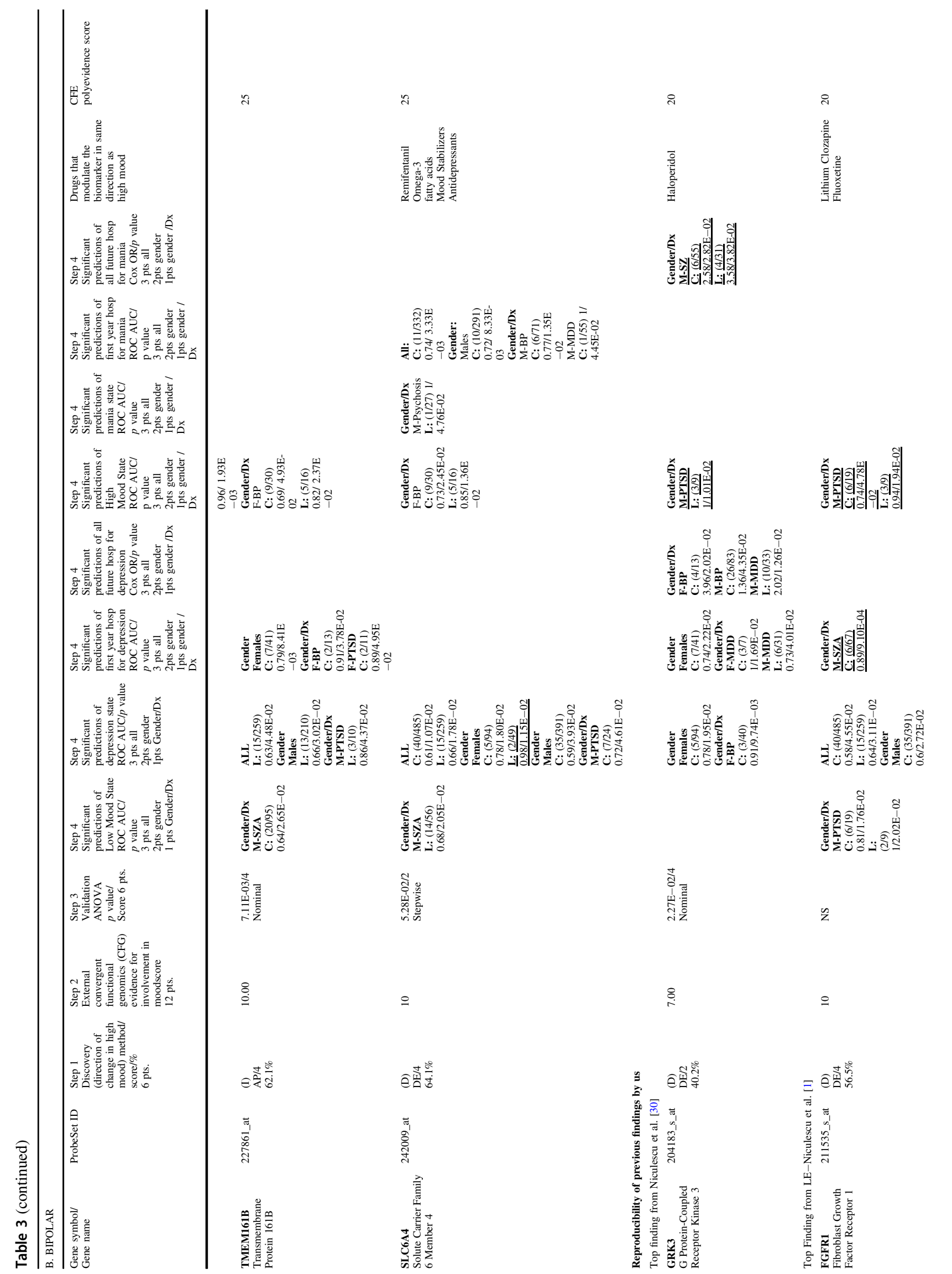




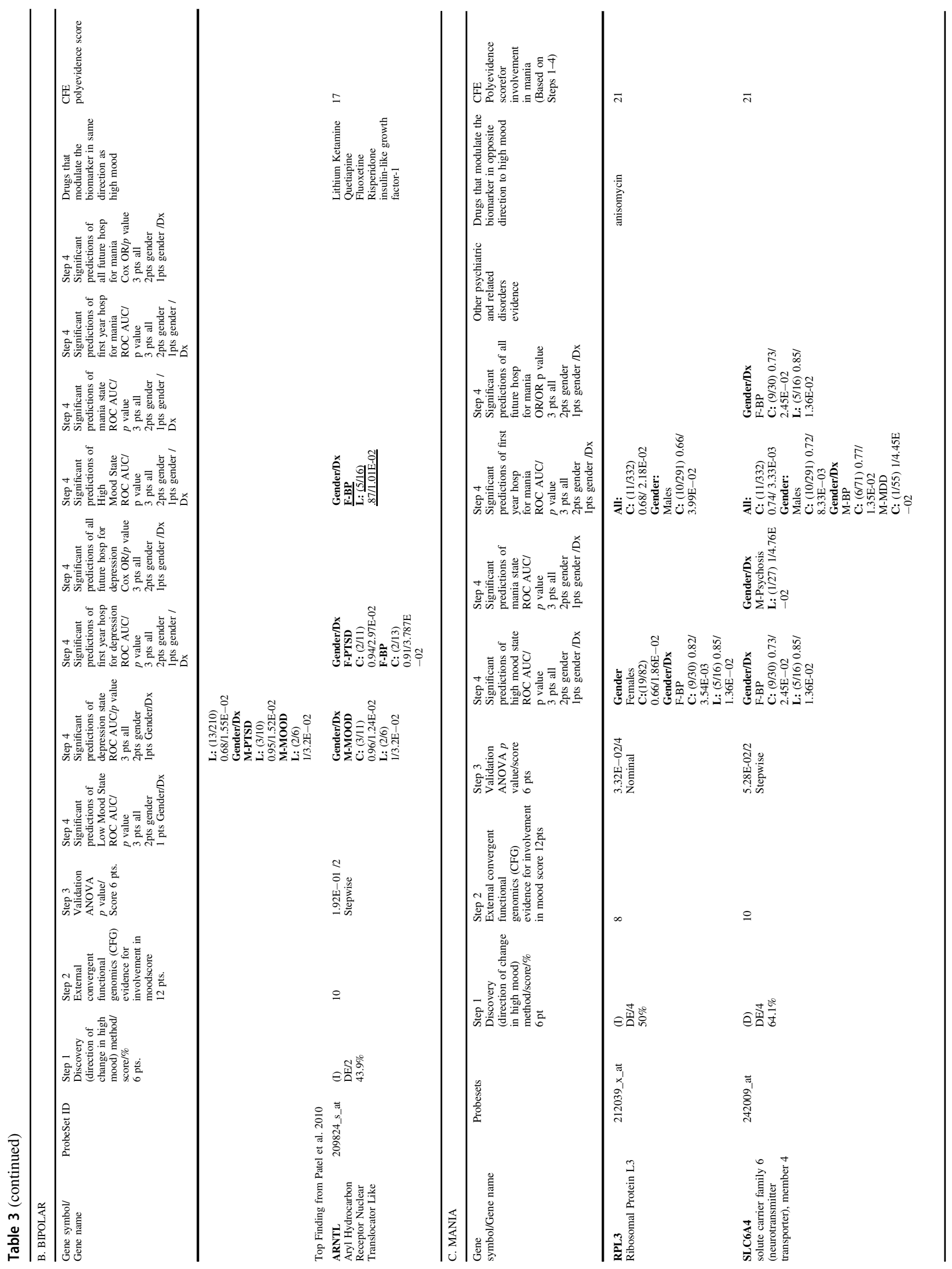




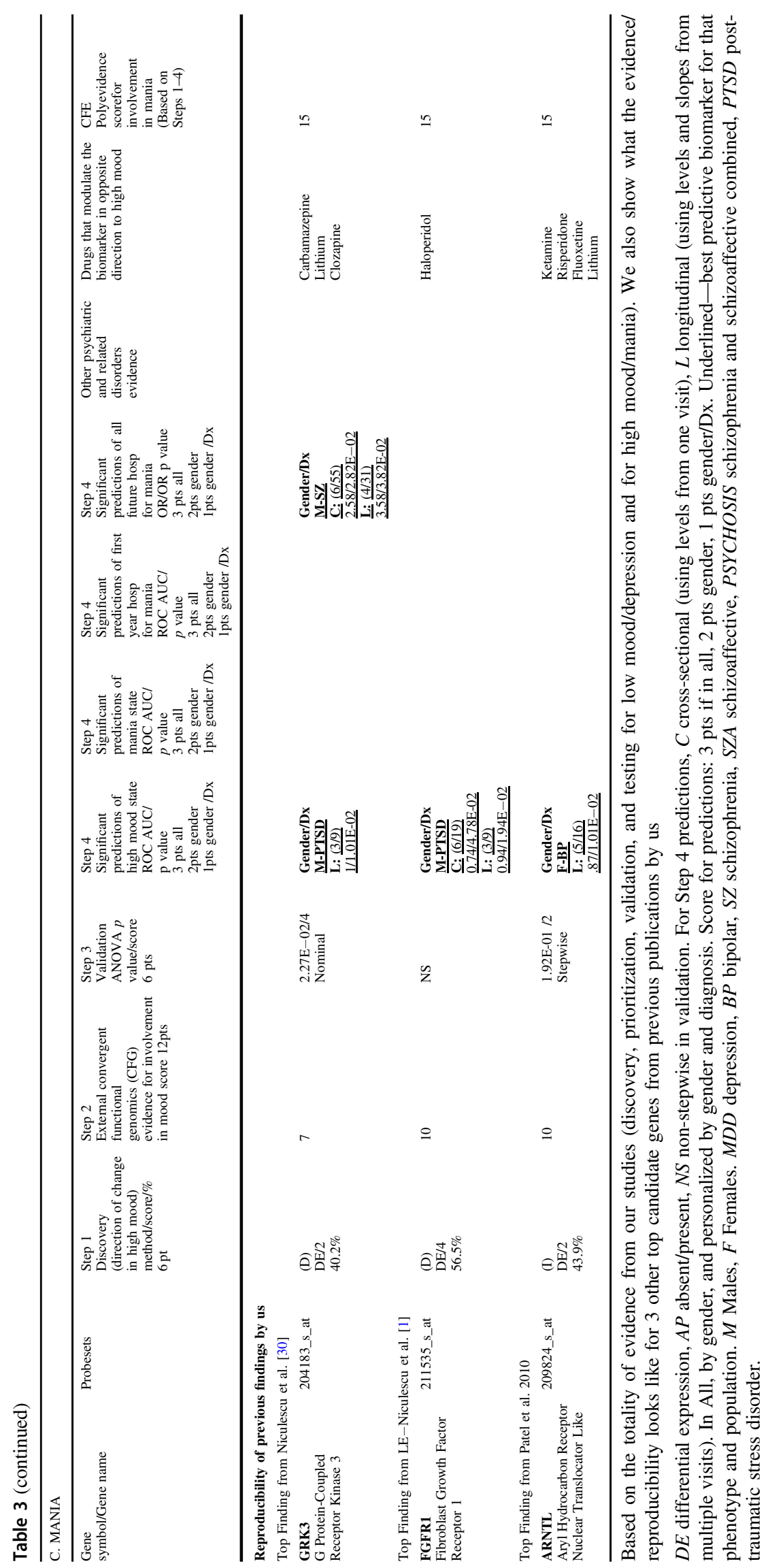


continuum in a quantitative fashion clinical symptoms for depression and mania, and provides a score for mood state at a particular moment in time. This is a state measure, related to how people feel in the present. It has good face validity based on DSM criteria, and correlates inversely with HAMD [12] (Fig. S1B). SASS, in addition to seven items measuring mood (SMS-7), also has four items measuring anxiety (SAS-4). We also used the PANSS Positive scale, that measures positive psychotic symptoms. These last two measures (SAS-4 and PANSS Positive) may define subtypes of low mood, as shown in the Discovery cohort (Fig. S1E).

We also created and used a checklist/measure of clinical severity of bipolar disorder, based on past history, called Convergent Functional Information for Bipolar Disorder Severity (CFI-BP) scale, ranking patients with mood disorders on a scale of $1-10$. This is a trait measure, related to how people behaved in their past (Fig. S2).

At each visit, we collected whole blood $(5 \mathrm{ml})$ in two RNA-stabilizing PAXgene tubes, labeled with an anonymized study ID number, and stored at $-80^{\circ} \mathrm{C}$ in a locked freezer until the time of future processing. Whole-blood RNA was extracted for microarray gene expression studies from the PAXgene tubes, as detailed below.

For this study, our within-subject discovery cohort, from which the biomarker data were derived, consisted of 44 subjects (30 males, 14 females) with psychiatric disorders and multiple testing visits, who each had at least one diametric change in SMS-7 mood scores from low mood (SMS-7 $\leq 40$ ) to high mood (SMS-7 $\geq 60$ ), or vice versa, from one testing visit to another. There were 4 subjects with 6 visits each, 6 subjects with 4 visits each, 18 subjects with 3 visits each, and 16 subjects with 2 visits each resulting in a total of 134 blood samples for subsequent gene expression microarray studies (Fig. 1, Tables 1 and S1).

Our independent validation cohort, in which the top biomarker findings were validated for being even more changed in expression, consisted of 39 male and 8 female subjects with a clinically severe mood disorder $(n=30$ depression as measured by HAMD scores $\geq 22$, and $n=17$ mania as measured by YMRS scores $\geq 20$ ), and concordant low mood, respectively high mood, SMS-7 scores (Tables 1 and S1).

Our independent test cohort for predicting low-mood state (SMS-7 $\leq 40$ ) and high-mood state (SMS-7 $\geq 60$ ) consisted of 153 male and 37 female subjects with psychiatric disorders, demographically matched with the discovery cohort, with one or multiple testing visits in our study, with either low mood, intermediate mood, or high mood states (Fig. 1 and Table 1).

Our independent test cohort for predicting clinical depression state $(H A M D \geq 22)$ consisted of 181 male and 45 female subjects with psychiatric disorders, demographically matched for age, with one or multiple testing visits in our study, with either low, intermediate, or high HAMD scores. Our independent test cohort for predicting clinical mania state (YMRS $\geq 20$ ) consisted of 73 males and 24 female subjects with psychiatric disorders, demographically matched for age, with one or multiple testing visits in our study, with either low, intermediate, or high YMRS scores (Fig. 1 and Table 1).

Our test cohorts for predicting future hospitalizations with depression, and future hospitalizations with mania (Fig. 1 and Table 1), are a subset of the independent test cohort for which we had longitudinal follow-up with electronic medical records. The subjects' subsequent number of hospitalizations with depression, and with mania, was tabulated from electronic medical records.

\section{Medications}

The subjects in the discovery cohort were all diagnosed with various psychiatric disorders (Table 1), and had various medical co-morbidities. Their medications were listed in their electronic medical records, and documented by us at the time of each testing visit. Medications can have a strong influence on gene expression. However, there was no consistent pattern of any particular type of medication, as our subjects were on a wide variety of different medications, psychiatric and non-psychiatric. Furthermore, the independent validation and testing cohort's gene expression data were $Z$-scored by gender and by diagnosis before being combined, to normalize for any such effects. Some subjects may be non-compliant with their treatment and may thus have changes in medications or drug of abuse not reflected in their medical records. That being said, our goal is to find biomarkers that track mood, regardless if the reason for it is endogenous biology or it is driven by medications or drugs. In fact, one would expect some of these biomarkers to be targets of medications, as we show in this paper. Moreover, the prioritization step that occurs after discovery is based on a field-wide convergence with literature that includes genetic data and animal model data, that are unrelated to medication effects. Overall, the discovery, validation, and replication by testing in independent cohorts of the biomarkers, with our design, occurs despite the subjects having different genders, diagnoses, being on various different medications, and other lifestyle variables.

\section{Blood gene expression experiments}

\section{RNA extraction}

Whole blood $(2.5 \mathrm{ml})$ was collected into each PaxGene tube by routine venipuncture. PaxGene tubes contain proprietary reagents for the stabilization of RNA. RNA was extracted and processed as previously described [3-5]. 


\section{Microarrays}

Microarray work was carried out using previously described methodology [3-6].

Of note, all genomic data were normalized (RMA for technical variability, then $z$-scoring for biological variability), by gender and psychiatric diagnosis, before being combined and analyzed.

\section{Biomarkers}

\section{Step 1: Discovery}

We have used the subject's score from a visual-analog scale (SMS-7), assessed at the time of blood collection (Fig. 1). We analyzed gene expression differences between visits with low mood (defined as a score of 0-40) and visits with high mood (defined as a score of 60-100), using a powerful withinsubject design, then an across-subjects summation (Fig. 1).

We analyzed the data in two ways: an absent-present (AP) approach, and a differential expression (DE) approach, as in previous work by us on suicide biomarkers [3-5]. The AP approach may capture turning on and off of genes, and the DE approach may capture gradual changes in expression. Analyses were performed as previously described [4-6]. In brief, we imported all Affymetrix microarray data as CEL. files into Partek Genomic Suites 6.6 software package (Partek Incorporated, St Louis, MI, USA). Using only the perfect match values, we ran a robust multi-array analysis (RMA) by gender and diagnosis, background corrected with quantile normalization and a median polish probeset summarization of all chips, to obtain the normalized expression levels of all probesets for each chip. Then, to establish a list of differentially expressed probesets we conducted a within-subject analysis, using a fold change in expression of at least 1.2 between consecutive high- and low-mood visits within each subject. Probesets that have a 1.2-fold change are then assigned either a 1 (increased in high mood) or a -1 (decreased in high mood) in each comparison. Fold changes between 1.1 and 1.2 are given 0.5 , and fold changes less than 1.1 are given 0 . These values were then summed for each probeset across all the comparisons and subjects, yielding a range of raw scores. The probesets above the $33.3 \%$ of raw scores were carried forward in analyses (Fig. 1), and received an internal score of 2 points; those above $50 \% 4$ points, and those above $80 \% 6$ points [4$6]$. We have developed in our labs $\mathrm{R}$ scripts to automate and conduct all these large dataset analyses in bulk, checked against human manual scoring [6].

Gene Symbol for the probesets were identified using NetAffyx (Affymetrix) for Affymetrix HG-U133 Plus 2.0 GeneChips, followed by GeneCards to confirm the primary gene symbol. In addition, for those probesets that were not assigned a gene symbol by NetAffyx, we used GeneAnnot (https://genecards.weizmann.ac.il/geneannot/index.shtml), or if need be UCSC (https://genome.ucsc.edu), to obtain gene symbol for these uncharacterized probesets, followed by GeneCard. Genes were then scored using our manually curated convergent functional genomics (CFG) databases as described below (Fig. 1D).

\section{Step 2: Prioritization using CFG}

Databases We have established in our laboratory (Laboratory of Neurophenomics, www.neurophenomics.info) manually curated databases of the human gene expression/protein expression studies (postmortem brain, peripheral tissue/fluids: CSF, blood and cell cultures), human genetic studies (association, copy number variations and linkage), and animal model gene expression and genetic studies, published to date on psychiatric disorders. Only findings deemed significant in the primary publication, by the study authors, using their particular experimental design and thresholds, are included in our databases. Our databases include only primary literature data and do not include review papers or other secondary data integration analyses to avoid redundancy and circularity. We also favored unbiased discovery studies over candidate genes hypothesis-driven studies. These large and constantly updated databases have been used in our CFG cross validation and prioritization platform (Fig. 1D). For this study, data from 1600 papers on mood disorders were present in the databases at the time of the CFG for mood disorders analyses (June 2018) (human genetic studies-759, human brain studies-246, human peripheral tissue/fluids- 359, non-human genetic studies-47, non-human brain/studies-167, non-human peripheral tissue/fluids- 22). We have developed in our lab a computerized CFG Wizard to automate and score in bulk large lists of genes by integrating evidence from these large databases, checked against manual scoring [6]. Analyses were performed as previously described $[4,5]$.

\section{Step 3: Validation analyses}

We examined which of the top candidate genes (score of 6 or above after the first two steps) were stepwise changed in expression from the clinically depressed validation group to the low-mood discovery group to the high-mood discovery group to the clinically manic validation group. A total score of 6 or above after the first two steps permits the inclusion of potentially novel genes with maximal internal score of 6 from discovery but no external evidence CFG score from prioritization. Subjects with low mood as well as subjects with high mood from the discovery cohort who did not have clinically severe depression or mania were used, along with the independent validation cohort $(n=47)$.

The AP-derived and DE-derived lists of genes were combined, and the gene expression data corresponding to 
them was used for the validation analysis. The four groups (clinical depression, low mood, high mood, clinical mania) were assembled out of Affymetrix.cel data that were RMA normalized by gender and diagnosis. We transferred the log transformed expression data to an Excel sheet, and non-log transformed the data by taking 2 to the power of the transformed expression value. We then $Z$-scored the values by gender and diagnosis. We then imported the excel sheets with the $Z$-scored by gender and diagnosis expression data into Partek, and statistical analyses were performed using a one-way ANOVA for the stepwise changed probesets, and also did a stringent Bonferroni correction for all the probesets tested in ANOVA (Fig. 1E).

\section{Top candidate biomarkers (after the first 3 steps)}

Adding the scores from the first three steps into an overall convergent functional evidence (CFE) score (Fig. 1), we ended up with a list of 26 top candidate biomarkers (26 probesets in $n=23$ genes), that had evidence, i.e., a CFE score, as good as or better than SLC6A4 (the serotonin transporter) (see also Supplementary Information- Pathways, Predictions and Reproducibility). SLC6A4 is arguably the most well studied molecular underpinning of mood disorders in biological psychiatry, and the target of the majority of antidepressant medications. We discovered it empirically as a blood biomarker as part of our work, and used it as a de facto positive control and cutoff. These 26 top candidate biomarkers were carried forward into additional analyses for biological understanding and for clinical utility.

\section{Biological understanding}

\section{Clock gene database}

We compiled a database of genes associated with circadian function, by using a combination of review papers [13, 14] and searches of existing databases CircaDB (http://circadb. hogeneschlab.org), GeneCards (http://www.genecards.org), and GenAtlas (http://genatlas.medecine.univ-paris5.fr). Using the data we compiled from these sources we identified a total of 1468 genes that show circadian functioning. We further classified genes into "core" clock genes, i.e., those genes that are the main engine driving circadian function $(n=18)$, "immediate" clock genes, i.e., the genes that directly input or output to the core clock $(n=331)$, and "distant" clock genes, i.e., genes that directly input or output to the immediate clock genes $(n=1119)$.

\section{Pathway analyses}

IPA (Ingenuity Pathway Analysis, version 24390178, Qiagen), David Functional Annotation Bioinformatics
Microarray Analysis (National Institute of Allergy and Infectious Diseases) version 6.7 (August 2016), and Kyoto Encyclopedia of Genes and Genomes (KEGG) (through DAVID) were used to analyze the biological roles, including top canonical pathways and diseases (Table 2). We performed the pathway analyses for the 26 biomarkers (23 unique genes) that were the top candidate biomarkers after the discovery, prioritization, and validation.

\section{Networks}

For network analyses we performed STRING Interaction network (https://string-db.org) by inputting the genes into the search window, and performed Multiple Proteins Homo sapiens analysis (Fig. S3).

\section{CFG beyond mood: evidence for involvement in other psychiatric and related disorders}

We also used a CFG approach to examine evidence from other psychiatric and related disorders, as exemplified for the list of top biomarkers after Step 4 testing (Table S3). This was not used to prioritize genes, but rather to understand the molecular basis of clinical co-morbidities.

\section{Testing for clinical utility in independent cohorts}

We tested in independent cohorts of psychiatric patients the ability of each of the top candidate biomarkers $(n=26)$ to assess state severity (mood (measured by SMS-7), depression (measured by HAMD), mania (measured by YMRS)), and predict trait risk (future hospitalizations with depression, future hospitalizations with mania). We conducted our analyses across all patients, as well as personalized by gender and diagnosis. We then predict with the biomarkers from the list in independent cohorts state (low-mood SMS$7 \leq 40$, depression HAMD $\geq 22$ ), and trait (Future Hospitalizations with Depression) in the first year of follow-up, and in all future years of follow-up. We also conducted similar analyses for predicting high mood, mania, and future hospitalizations for mania.

The test cohort for predicting low mood/depression(state), and the test cohort for predicting future Hospitalizations with Depression (trait), was assembled out of data that were RMA normalized by gender and diagnosis. The cohort was completely independent from the discovery and validation cohorts, there was no subject overlap with them. Individual markers used for predictions were $Z$-scored by gender and diagnosis, to be able to combine different biomarkers into panels and to avoid potential artefacts due to different ranges of expression in different gender and diagnoses. For panels, biomarkers were combined by simple summation of the increased risk biomarkers minus the decreased risk 
biomarkers. Predictions were performed using R-studio. For cross-sectional analyses, we used biomarker expression levels, $z$-scored by gender and diagnosis. For longitudinal analyses, we combined four measures: biomarker expression levels, slope (defined as ratio of levels at current testing visit vs. previous visit, divided by time between visits), maximum levels (at any of the current or past visits), and maximum slope (between any adjacent current or past visits). For decreased biomarkers, we used the minimum rather than the maximum for level calculations. All four measures were $Z$-scored, then combined in an additive fashion into a single measure. The longitudinal analysis was carried out in a subcohort of the testing cohort consisting of subjects that had at least two visits (timepoints).

\section{Predicting state- low mood, clinical depression}

Receiver-operating characteristic (ROC) analyses between marker levels and mood state were performed by assigning subjects visits with a mood SMS-7 score of $\leq 40$ into the low mood category, and subjects with HAMD scores $\geq 22$ in the clinically depressed category. We used the pROC package of R (Xavier Robin et al. BMC Bioinformatics 2011). (Table 3 and Fig. 2). In addition, a one-tailed t-test was performed between low mood group vs. the rest, and Pearson R (one-tail) was calculated between mood scores and biomarker levels.

Similar analyses were conducted for high mood state (SMS-7 score of $\geq 60$ ) and clinical mania state (YMRS $\geq 20$ ).

\section{Predicting trait- future psychiatric hospitalization with depression as a symptom/reason for admission}

We conducted analyses for predicting future psychiatric hospitalizations with depression as a symptom/reason for admission in the first year following each testing visit, in subjects that had at least 1 year of follow-up in the VA system, in which we have access to complete electronic medical records. ROC analyses between biomarkers measures (cross-sectional, longitudinal) at a specific testing visit and future hospitalizations were performed as described above, based on assigning if subjects had been admitted to the hospital with depression or not. In addition, a one tailed t-test with unequal variance was performed between groups of subject visits with and without future hospitalization with depression. Pearson R (one-tail) correlation was performed between hospitalization frequency (number of hospitalizations with depression divided by duration of follow-up) and marker levels. A Cox regression was performed using the time in days from the testing visit date to first hospitalization date in the case of patients who had been hospitalized, or 365 days for those who did not. The odds ratio (OR) was calculated such that a value greater than 1 always indicates increased risk for hospitalization, regardless if the biomarker is increased or decreased in expression.

We also conducted Cox regression and Pearson $R$ analyses for all future hospitalizations with depression, including those occurring beyond 1 year of follow-up, in the years following testing (on average 5.12 years per subject, range $0.07-11.27$ years), as these calculations, unlike the ROC and t-test, account for the actual length of follow-up, which varied from subject to subject. The ROC and $t$-test might in fact, if used, under-represent the power of the markers to predict, as the more severe psychiatric patients are more likely to move geographically and/or be lost to follow-up. The Cox regression was performed using the time in days from visit date to first hospitalization date in the case of patients who had hospitalizations with depression, or from visit date to last note date in the electronic medical records for those who did not.

Similar analyses were conducted for future hospitalizations with mania as a symptom/reason for hospitalization.

\section{Therapeutics}

\section{Pharmacogenomics}

We analyzed which of the top biomarkers for depression and for mania after Steps 1-4 are known to be changed in expression by existing drugs in a direction opposite to the one in disease, using our CFG databases, and using Ingenuity Drugs analyses (Table 3 and Table S4).

\section{New drug discovery/repurposing}

We also analyzed which drugs and natural compounds are an opposite match for the gene expression signatures of our top biomarkers, using the Connectivity Map (https://portals. broadinstitute.org, Broad Institute, MIT) (Fig. 3 and Table 4). Of note, not all the probesets from the HG-U133 Plus 2.0 array we used were present in the HGU-133A array used for the Connectivity Map. We stayed with exact probeset level matches, not gene level imputation. We also used the NIH LINCS database to conduct similar analyses, at a gene level.

\section{Report generation}

We present an example of how a report to doctors might look, using the above insights. We used a panel of top biomarkers after Steps 1-4 (Fig. 3 and Table 3): BioM12 + 1: $n=12$ genes to generate a score for depression severity, as well as the mania biomarker RLP3 to inform risk for bipolar switching. Out of a dataset of 794 subject visits, we chose as a case study a visit from a 
A.

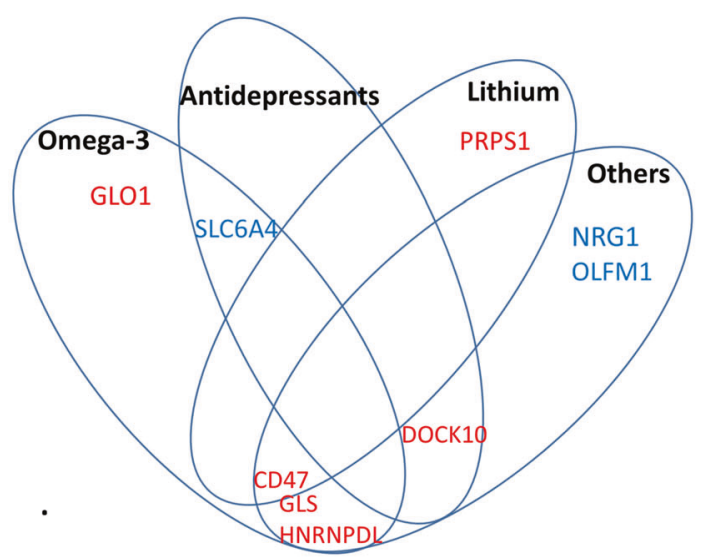

Fig. 3 Therapeutics: matching with medications. A Pharmacogenomics. See also Tables 3 and S4. B New repurposed drugs using the panels of markers. See also Table 4. There is overlap between depression, bipolar and mania biomarkers. RPL3 could be targeted to treat mania with less risk of inducing depression. Six biomarkers (CD47, FANCF, GLO1, HNRNPDL, OLFM1, SMAD7) could be targeted to treat depression with less risk of inducing mania. Other six

female patient with depression who had died by suicide, a case previously discussed in a suicide biomarker paper of ours (Levey et al. [5]) (Fig. 4).

First, we removed that patient from the dataset, and divided the remaining dataset into three populations: those who had a high HAMD score $\geq 22$ (concordant with a low SMS-7 mood score $\leq 40$ ), those who had a low HAMD score $\leq 7$, and those who had an intermediate HAMD score. For the first two groups, we calculated the average $Z$-scored expression values for each biomarker in the panel. We then compared the levels of each biomarker, in each subject in the cohort, including the subject of interest, to these reference levels. If a biomarker was higher than the average of the high HAMD subjects it got a 1 , if it was below the average of the low HAMD subjects it got a 0 , and if it was in between it got a 0.5 . Next, we averaged the biomarkers in the panel and multiplied by 100 , to generate a score between 0 and 100 for the BioM12 for each of the 794 subjects, including the case study subject. This digitalization of the scores was done to avoid overfitting to our particular cohort, and provide an easily understandable and interpretable readout for clinicians. The score of the BioM12 is compared to the average score of BioM12 for the high HAMD subjects and the low HAMD subjects, generating 3 risk categories: high (red), intermediate (yellow), and low (green) for current depression severity. This rank percentile of the score of the patient compared to the

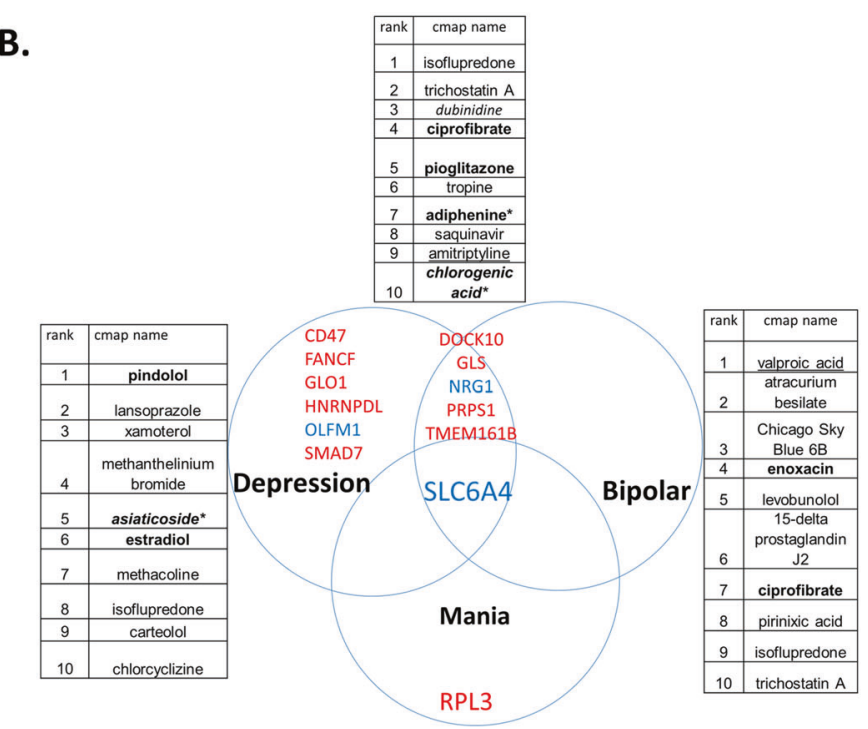

biomarkers (DOCK10, GLS, NRG1, PRPS1, TMEM161B, SLC6A4) could be targeted to treat depression fast/powerfully, but may induce mania, so need to be coupled with a mood stabilizer or antipsychotic. An example of the latter is SLC6A4. SSRIs should thus be used cautiously in monotherapy to treat depression, and clinicians should have a low threshold for adding mood stabilizers.

distribution of scores of subjects in the database is also provided in the report (Fig. 4).

Second, future risk is assessed by looking how many of three biomarkers in the panel, that are good predictors of future hospitalizations for depression (NRG1, PRPS1, SMAD7), were a 1 , generating 0 to 3 asterisks.

Third, we examined how many of the bipolar biomarkers $(n=6)$ in BioM12 were a score of 1 . If more than $50 \%$ of them (more than 3 out of 6 ) were a 1 , than the patient gets an asterisk for bipolar risk. If the mania biomarker RLP3 is also 1 , then the patient gets another asterisk for risk of bipolarity, i.e., risk of switch if treated for depression. In those with asterisks for risk of bipolarity, it is advisable to choose mood stabilizers or antipsychotics from the medication choices provided by the report.

Fourth, for each biomarker in the panel, we also have a list of existing psychiatric medications that modulate the expression of the biomarker in the direction of high mood. Each medication got a score of 1 or 0 whether it modulated a particular biomarker in the panel or not, and that score is multiplied with the risk score of the biomarker, i.e., 1 or 0.5 or 0 . A medication can modulate more than one biomarker. We then calculated an average score for each medication based on its effects on all the biomarkers in the panel, and multiplied that by 100 , resulting in a score of 0 to 100 for each medication. Thus, psychiatric medications are matched to the patient and ranked in order of impact on the panel. 
Table 4 Therapeutics: drug repurposing for depression.

A. Connectivity Map (CMAP) analyses

Rank CMAP name Score Role

A1. Drugs identified using gene expression panels of biomarkers with highest evidence (CFE) for involvement in depression (BioM12 depression-12 genes-NRG1, PRPS1, GLS, DOCK10, TMEM161B, GLO1, HNRNPDL, FANCF, CD47, SMAD7, OLFM1, SLC6A4). See Table 3A and Fig. 3. Direction of expression in high mood (CMAP).

1 Isoflupredone 1 Synthetic glucocorticoid that may be considered as an alternative to traditional corticosteroids. Isoflupredone is the only corticosteroid approved by the U.S. Food and Drug Administration for use exclusively in large animals, including lactating cattle.

2 Trichostatin A 0.963 HDAC inhibitor

3 Dubinidine 0.943 Anticonvulsant which reduces motor activity, enhances the effects of alcohol, ether and barbiturates. Quinoline alkaloid, from plants of the Rutaceae Family.

4 Ciprofibrate 0.939 PPAR-alpha activator, lipid lowering agent

5 Pioglitazone 0.931 PPAR- $\gamma$ activator, anti-diabetic (*also in our work on Alzheimer [10])

6 tropine 0.93 Alkaloid

7 Adiphenine* 0.907 Anticholinergic, antispasmodic (*also in our work on suicidality [6])

8 Saquinavir 0.903 Anti-retroviral medication

9 Amitriptyline 0.902 Tricyclic antidepressant.

10 Chlorogenic 0.897 Antioxidant, polyphenol found in coffee (*also in our work on suicidality [6]) acid*

A2. Drugs identified using gene expression panels of biomarkers with highest evidence (CFE) for involvement in depression specific without overlap with bipolar (BioM6 Depression-specific-6 genes-GLO1, HNRNPDL, FANCF, CD47, SMAD7, OLFM1). Direction of expression in high mood (CMAP). See Fig. 3.
1 Pindolol
$1 \quad \beta$-blocker, and is also a potent serotonin $5 \mathrm{HT}_{1 \mathrm{~A}}$ presynaptic receptor antagonist
2 Lansoprazole
0.977 Proton pump inhibitor (PPI), that works by decreasing the amount of acid produced by the stomach.
3 Xamoterol
0.975 Cardiac stimulant, that works by binding to the $\beta_{1}$ adrenergic receptor. It is a 3rd generation adrenergic $\beta$ receptor partial agonist. It provides cardiac stimulation at rest but it acts as a blocker during exercise.
4 Methanthelinium bromide
0.953 Muscarinic receptor antagonist (anticholinergic, parasympatholytic agent). Spasmolytic agent. Gastric acid secretion inhibitor.
5 Asiaticoside*
0.927 Triterpenoid component derived from Centella asiatica (L.) and widely used in antioxidant, anti-inflammatory, immunomodulatory, and wound healing applications. (*also in our work on suicidality [6])
6 Estradiol
0.924 Female sex hormone
7 Methacholine
0.923 Muscarinic agonist
8 Isoflupredone
0.916 Steroid
9 Carteolol
0.913 Beta blocker
10 Chlorcyclizine
0.911 First-generation antihistamine. It is used primarily to treat allergy symptoms such as rhinitis, urticaria, and pruritus, and may also be used as an antiemetic.

\footnotetext{
A3. Drugs identified using gene expression panels of biomarkers overlapping between depression and bipolar (BioM6 bipolar depression -6 genes-NRG1, DOCK10, GLS, PRPS1, TMEM161B, and SLC6A4). Direction of expression in high mood. (CMAP). See Table 3B and Fig. 3.

1 Valproic acid 1 HDAC inhibitor, mood stabilizer

2 Atracurium besilate 0.991 Nicotinic antagonist muscle relaxant

3 Chicago Sky 0.98 Histological stain that also is a vesicular glutamate transporters inhibitor, attenuating methamphetamine-induced Blue 6B hyperactivity and behavioral sensitization in animal models

4 Enoxacin

0.972 Fluoroquinolone antibiotic that also elevates microRNA levels and prevents learned helplessness in animal models

5 Levobunolol

0.969 Beta-blocker

6 15-delta prostaglandin $\mathrm{J} 2$

0.95 Anti-inflammatory lipid mediator and PPAR- $\gamma$ activator. It is made from prostaglandin D2. Decreased Prostaglandin D2 Levels in Major Depressive Disorder Are Associated with Depression-Like Behaviors in human and animal model studies.

7 Ciprofibrate $\quad 0.949$ PPAR-alpha activator, lipid lowering agent
} 
Table 4 (continued)
8 Pirinixic acid
0.949 PPAR-alpha activator, anti-lipid agent
9 Isoflupredone
0.947 Synthetic glucocorticoid
10 Trichostatin A
0.946 HDAC inhibitor

B. NIH LINCS L1000 characteristic direction signature search engine analyses

\begin{tabular}{|c|c|c|c|}
\hline Rank & Score & Drug & Description \\
\hline \multicolumn{4}{|c|}{$\begin{array}{l}\text { B1. Drugs identified using gene expression panels of biomarkers with highest evidence (CFE) for involvement in depression (BioM12 } \\
\text { Depression- } 12 \text { genes). See Table } 3 \mathrm{~A} \text { and Fig. 3. Direction of expression in high mood ( } 9 \text { increased and } 3 \text { decreased). }\end{array}$} \\
\hline 1 & 0.3 & NNC 55-0396 dihydrochloride & T-type calcium channel blocker \\
\hline 2 & 0.3 & Nadolol & Beta blocker \\
\hline 3 & 0.3 & MLN4924 & Inhibitor of Nedd8-Activating Enzyme \\
\hline 4 & 0.2 & U0126 & MEK $1 / 2$ inhibitor \\
\hline 5 & 0.2 & Nortryptiline & Tricyclic antidepressant \\
\hline 6 & 0.2 & Amcinonide & Synthetic glucocorticoid \\
\hline 7 & 0.2 & Iopanic acid & Iodine-containing radiocontrast medium, thyroid inhibitor \\
\hline 8 & 0.2 & $\underline{\text { Paroxetine }}$ & SSRI antidepressant \\
\hline 9 & 0.2 & Rosuvastatin & Statin \\
\hline 10 & 0.2 & trichostatin $\mathrm{A}$ & HDAC inhibitor \\
\hline
\end{tabular}

Drugs that have opposite gene expression effects to the gene expression signature of our nominally significant predictive biomarkers for depression(A1-A2) and for bipolar depression(A3), using the Connectivity Map [36] (CMAP), and for depression (B1) using the NIH LINCS database. Bold—new drugs of immediate interest. Italic — natural compound. Underlined—known drugs that serve as a de facto positive control.

Fifth, we used the biomarkers that were positive as high risk in the panel, to interrogate the CMAP and do individualized drug repurposing, identifying new non-psychiatric compounds that could be used in that particular patient to treat depression (Fig. 4).

\section{Results}

In Step 1 Discovery, we identified candidate blood gene expression biomarkers that: (1) change in expression in blood between self-reported low-mood and high-mood states, (2) track the mood state across visits in a subject, and (3) track the mood state in multiple subjects. We used a visual analog measure for mood state (SMS-7). At a phenotypic level, the SMS-7 quantitates mood state at a particular moment in time, and normalizes mood measurements in each subject, comparing them to the lowest and highest mood that subject ever experienced. We then used a powerful within-subject and then across-subject design in a longitudinally followed cohort of subjects ( $n=44$ subjects, with 134 visits) who displayed at least a 50\% change in the mood measure (from below 40/100 to above 60/100) between at least two consecutive testing visits, to identify differentially expressed genes that track mood state. Using our $33 \%$ of maximum raw score threshold (internal score of 2 pt) $[4,5]$, we had 11,620 unique probesets (corresponding to 9649 unique genes) from Affymetrix Absent/Present
(AP) analyses and DE analyses (Fig. 1D). These were carried forward to the prioritization step. This represents approximately a fivefold enrichment of the 54,625 probesets on the Affymetrix array.

We also examined in the discovery cohort whether subtypes of low mood can be identified based on mental state at the time of low mood visits, using two-way hierarchical clustering with anxiety and psychosis measures. The mood state self-report may be more reliable in this cohort, as the subjects demonstrated the aptitude and willingness to report different, and diametric, mood states. We uncovered four potential subtypes of low mood/depression: high anxiety and low psychosis (anxious), high anxiety and high psychosis (combined), low anxiety and high psychosis (psychotic), low anxiety and low psychosis (pure low mood) (Fig. S3). These subtypes need to be tested in future studies in independent cohorts for practical utility, diagnostic and therapeutic.

In Step 2 Prioritization, we used a CFG approach to prioritize the candidate biomarkers identified in the discovery step ( $33 \%$ cutoff, internal score of $\geq 2$ pt.) by using published literature evidence (genetic, gene expression and proteomic), from human and animal model studies, for involvement in mood disorders (Fig. 1E and Table S2). There were 6370 probesets (corresponding to 4960 unique genes) that had a total score (combined discovery score and prioritization CFG score) of 6 and above. These were carried forward to the validation step. This represents 
Phchp328v1

Female, MDD, 37 years old, Caucasian

$\mathrm{HAMD}=25$

SMS7 $=17.4 / 100$

\section{Depression Score}

\author{
$88.46(89.9 \%)$ \\ High \\ Future Risk for Depression: $(* * *)$ High \\ Risk for Bipolar Switching: (**) High \\ Consider using mood stabilizers and antipsychotics \\ rather than antidepressants
}

Top 5 List of Suggested Existing Psychiatric Medications :

\begin{tabular}{cc} 
Drugs & Percentile \\
\cline { 2 - 2 } Omega 3 & 39.1 \\
Lithium & 17.4 \\
Clozapine & 17.4 \\
Venlafaxine & 13 \\
Diazepam & 8.7
\end{tabular}

Top 5 List of Suggested New Non- Psychiatric Medications :

rank
1
2
3
4
5

\author{
New Drug \\ Methanthelinium bromide \\ isoflupredone
}

Pindolol

dubinidine

ciprofibrate

\section{Recommendations: \\ Omega-3, Lithium, Pindolol \\ Consider adding: \\ Clozapine, methanthelinium bromide (Vagantin, Germany)}

Fig. 4 Example of report for physicians. Using the panel of the top biomarkers BioM12 + 1: Depression ( $n=12$ genes), as well as RPL3 for mania risk. This subject (Phchp328) was previously described by us in a suicidality biomarker study (Levey et al. [5]), as high risk for suicide, and died by suicide a year after completing our study. No information was provided to her clinicians by us at that time due to anonymity and privacy rules in research studies. The raw expression values of the biomarkers were $Z$-scored by gender and diagnosis. The $Z$-scored expression value of each increased biomarker was compared to the average value for the biomarker in the severely depressed group $(H A M D \geq 22)$, and the average value of the non-depressed group (HAMD $\leq 7)$, resulting in scores of 1 or 0 , respectively, and 0.5 if it was in between. The reverse was done for decreased biomarkers. The "digitized" biomarkers were then added into a polygenic risk score. The subject had a BioM12 polygenic depression score of 88.46 , being at the $90 \%$ of the 794 subjects in our database. Three out of the three biomarkers for future risk for depression hospitalizations (NRG1,

approximately a tenfold enrichment of the probesets on the Affymetrix array.

In Step 3 Validation, we validated for change in clinically severe mood disorders (depression, mania) these prioritized biomarkers, in a demographically matched cohort of ( $n=30$ clinically severe depression, $\mathrm{n}=17$ clinically severe mania), by assessing which markers were stepwise changed in expression: from clinically severe depression in validation cohort, to low mood in discovery cohort, to high mood in discovery cohort, and to clinically severe mania in the validation cohort (Fig. 1). Four thousand six hundred thirty-three probesets were not stepwise changed, and 1737 were stepwise changed. Of these, 291 probesets (corresponding to 283 unique genes) were nominally significant. This represents approximately a 188-fold enrichment of the probesets on the Affymetrix array.
PRPS1, SMAD7) had a score of 1 in this patient (***). More than $50 \%$ of the 6 bipolar biomarkers that are part of the BioM12 (Table 3A and B) $(*)$, as well as the mania marker RPL3 (Table 3C) $(*)$, had a score of 1 in this patient, resulting in increased risk for bipolar switching (**). This subject's clinical diagnosis was major depressive disorder (MDD), but it is likely she had bipolar disorder. The "digitized" biomarkers were also used for matching with existing psychiatric medications. Biomarkers were matched based on our CFG databases with existing psychiatric medications that had effects on gene expression opposite to depression, in the direction of high mood. Each medication matched to a biomarker got a score of 1 that was then multiplied with the biomarker score of $1,0.5$ or 0 . The scores for the medications were added, and medications prioritized by this score. In addition, the signature of the biomarkers in the panel that had a score of 1 , and their direction of change, was used to interrogate the CMAP and LINCS databases for new repurposed medications that would treat depression in this patient.

Adding the scores from the first three steps into an overall CFE score (Fig. 1), we ended up with a list of 26 top candidate biomarkers ( $n=23$ genes, 26 probesets), that had a CFE score as good as or better than SLC6A4, which serves as a de facto positive control and which we decided to use as an empirical cutoff. This represents approximately an over 2000-fold enrichment of the probesets on the Affymetrix array.

The list of 23 genes (26 probesets) (Fig. 1) is composed of genes increased in expression in high mood (TMEM161B, GLO1, PRPS1, SMAD7, ANK3, OGT, CD47, GLS, TMEM106B, RPL3, FANCF, HNRNPDL, DOCK10, CALM1), and genes decreased in expression in high mood (NRG1, OLFM1, SPECC1, SORT1, TPH1, GSK3B, MARCKS, NR3C1, and SLC6A4). These 26 top candidate biomarkers were carried forward into analyses for understanding biological underpinnings. Last but not least, 
they were tested for predictive ability and clinical utility in additional independent cohorts.

\section{Biological understanding}

\section{Biological pathways}

We conducted biological pathway analyses using the top candidate biomarkers for mood $(n=23$ genes, 26 probesets), which suggest that circadian, neurotrophic, and cell differentiation functions are involved, along with serotonergic and glutamatergic signaling, supporting a view of mood as reflecting activity and growth (Table 2A). Reassuringly, depression, along with weight gain, were the top diseases identified by the pathway analyses using DAVID, and Ingenuity identified neurological and psychological disorders as the top diseases.

\section{Circadian}

A number of top candidate biomarkers identified by us have biological roles that are related to the circadian clock (8 out of 23 genes) (Supplementary Information- Pathways, Predictions and Reproducibility). Circadian clock abnormalities are related to mood disorders $[14,15]$.

\section{Networks and interactions}

We conducted STRING analyses of the top candidate biomarkers that revealed groups of interacting proteins. In particular, NR3C1 ((Nuclear Receptor Subfamily 3, Group C, Member 1 (Glucocorticoid Receptor)) is at the overlap of a network containing SLC6A4 and TPH1, and one centered on GSK3B that also contains OGT and CALM1 (Fig. S3). A third network includes CALM1, GLO1, and MARCKS. These networks may have biological significance and could be targeted therapeutically. The first network is involved in reactivity (serotonin and stress response), the second one in activity (energy metabolism and growth), and the third one in connectivity (calcium intracellular signaling).

\section{Testing for clinical utility}

In Step 4 Testing, we examined in completely independent cohorts from the ones used for discovery or validation whether the 26 top candidate biomarkers can assess lowmood state $(n=190$ subjects), depression state $(n=$ 226 subjects), as well as predict of future psychiatric hospitalizations due to depression ( $n=170$ subjects) (Fig. 2 and Table 3), using electronic medical records follow-up data of our study subjects (up to 11.27 years from initial visit at the time of the analyses) (Fig. 1, Table 1, and Table S1). The gene expression data in the test cohorts were normalized (Z-scored) across genders and various psychiatric diagnoses, before those different demographic groups were combined. We used biomarker levels information cross-sectionally, as well as expanded longitudinal information about biomarker levels at multiple visits, as predictors. We tested the biomarkers in all subjects in the independent test cohort, as well as in a more personalized fashion by gender and psychiatric diagnosis.

For low mood state assessment across all subjects in the independent test cohort, the best biomarker was NRG1, increased in expression in low mood, with an AUC of $62 \%$ $(p=6.8 \mathrm{E}-03)$, and $64 \%(p=3.5 \mathrm{E}-02)$ for assessing clinical depression state. NRG1 also had a Cox regression OR of $1.17(p=2.5 \mathrm{E}-02)$ for predicting all future hospitalizations for depression, and an AUC of $87 \%(p=1.1 \mathrm{E}$ -03 ) for predicting first year hospitalizations for depression in females. Moreover, in the opposite direction, for assessing high-mood state across all subjects, NRG1 has a modest AUC of $58 \%(p=1.4 \mathrm{E}-02)$, but is a robust predictor of all future hospitalizations for mania in patients with psychotic disorders (OR of $2.7(p=3.3 \mathrm{E}-02)$. Consistent with our findings, NRG1 (neuregulin 1) has prior evidence as a biomarker for mood disorders, increased in expression in blood in depression, and decreased in expression after antidepressant treatment [16]. Interestingly, it is increased in expression in blood in our previous biomarker studies on suicidality [6], stress [9], pain [8], and psychosis [17], as well as increased in expression in blood in aging [18], all co-morbidities associated with depression. NRG1 is a membrane glycoprotein that mediates cell-cell signaling and plays a critical role in the activity, growth and development of multiple organ systems. It is a direct ligand for ERBB3 and ERBB4 tyrosine kinase receptors, resulting in ligand-stimulated tyrosine phosphorylation and activation of the ERBB receptors. Activity and trophicity of tissues may be key functions of mood [19].

For assessment of clinical depression state in the independent test cohort, DOCK10, decreased in expression in low mood, had an AUC of $73 \%(p=1.17 \mathrm{E}-03)$ across all subjects, and $75 \%(\mathrm{p}=1.05 \mathrm{E}-03)$ in males, surviving Bonferroni correction for all 26 biomarkers tested. It also had an AUC of $95 \%$ ( $p=1.52 \mathrm{E}-02)$ for males with PTSD. DOCK10 had a Cox regression OR of $1.9(p=3.93 \mathrm{E}-02)$ for predicting all future hospitalizations for depression in females. Moreover, in the opposite direction, for assessing high mood state, it has an AUC of $70 \%$ in females $(p=$ $2.63 \mathrm{E}-02)$, and $100 \%(p=9.18 \mathrm{E}-04)$ in females with bipolar disorder (Table 3). DOCK10 (dedicator of cytokinesis 10) has some prior human evidence in human blood from bipolars [20], and is decreased in expression in brain in an animal model of depression [21]. DOCK10 is also decreased in expression in human brains and blood in aging [18], as well as decreased in brain in an animal model of 
stress-induced depression, as described by Nestler and colleagues [22]. Interestingly, it is increased in expression in brain in animal models upon physical and cognitive stimulation [23]. There is human genetic evidence implicating this gene in longevity [24]. The link between depression, stress, aging and longevity is an area of active interest for our group $[25,26]$. DOCK10 is a guanine nucleotideexchange factor that activates $\mathrm{CDC} 42$ and $\mathrm{RAC} 1$ by exchanging bound GDP for free GTP. It is essential for dendritic spine morphogenesis in Purkinje cells and in hippocampal neurons, via a CDC42-mediated pathway.

SLC6A4 is an example of a previously well-known gene reproduced in this study. For clinical depression state assessment in the independent test cohort across all subjects, SLC6A4, increased in expression in low mood, had an AUC of $61 \%(p=1.1 \mathrm{E}-02)$ if measured cross-sectionally, and AUC of $66 \%(p=1.78 \mathrm{E}-02)$ if measured longitudinally. It has even better accuracy in females: an AUC of $78 \%(p=1.8 \mathrm{E}-02)$ if measured cross-sectionally, and an AUC of $98 \%(p=1.1 \mathrm{E}-02)$ if measured longitudinally. Moreover, in the opposite direction, for predicting future hospitalizations for mania in the first year, across all subjects, SLC6A4 had an AUC of 74\% ( $p=3.3 \mathrm{E}-03)$, and an even better accuracy in male bipolars, with an AUC of $77 \%$ $(p=1.3 \mathrm{E}-02)$. The product of this gene is the serotonin transporter, which is the target of serotonin reuptake inhibitors used to treat depression, as well as anxiety and stress disorders. Of note, it is known that individuals with bipolar disorder treated with SSRIs, especially in monotherapy, can switch into mania.

As exemplified above, we also conducted analyses looking at the ability of the 26 top candidate biomarkers to assess high mood/mania states, and predict future hospitalizations for mania (Table 3B,C, and see Supplementary Information-Pathways, Predictions and Reproducibility).

We also tested an algorithm combining as predictors BioM26, along with mood (SMS-7, Fig. S1) and with a measure of clinical severity of bipolar disorder (CFI-BP, Fig. S2), with modest synergistic effects (Table S1). Of note, CFI-BP was a good predictor of all future hospitalizations for mania in all (Cox regression OR of $2.9(p=2.5 \mathrm{E}$ $-04)$ ), and an even better predictor in males with bipolar disorder (OR of $3.2(p=8.3 \mathrm{E}-05))$.

\section{Convergent functional evidence (CFE)}

For the top candidate biomarkers $(n=26)$, we tabulated into a CFE score all the evidence from discovery (up to 6 points), CFG prioritization (up to 12 points), validation (up to 6 points), and testing (state low mood, state clinical depression, trait first year hospitalization with depression, trait all future hospitalizations with depression, as well as state high mood, state clinical mania, trait first year hospitalization with mania, trait all future hospitalizations with mania - up to three points each if it significantly predicts in all subjects, two points if in gender, one points if in gender/diagnosis). The total score can be up to 48 points: 36 from our empirical data, and 12 from literature data used for CFG. We weigh our new empirical data more than the literature data, as it is functionally related to mood in three independent cohorts (discovery, validation, and testing). The goal is to highlight, based on the totality of our data and of the evidence in the field to date, biomarkers that have all around evidence: track mood, have convergent evidence for involvement in mood disorders, and predict mood state and future clinical events (Table 3). Such biomarkers merit priority evaluation in future clinical trials.

The 6 top blood biomarkers with the strongest overall CFE for tracking and predicting both depression and mania, hence bipolar mood disorders, after the first four steps were NRG1, DOCK10, GLS, PRPS1, TMEM161B, and SLC6A4 (Table 3B). For example, NRG1 (neuregulin 1) decreased in expression in high mood, survived discovery, prioritization and validation. It seems to be a better predictor for low mood/depression, especially personalized by gender and diagnosis, than for high mood/mania (Table 3).

\section{Targeted therapeutics}

\section{Pharmacogenomics}

A number of individual top biomarkers are known to be modulated by medications in current clinical use for treating depression, such as by lithium (NRG1, PRPS1, CD47), antidepressants (SLC6A4, DOCK10, NRG1, CD47) and the nutraceutical omega-3 fatty acids (GLO1, SLC6A4, CD47, GLS, HNRNPDL) (Fig. S4 and Table 3 and S4). In particular, NRG1 is at the overlap of lithium and antidepressants, and CD47, which is involved in cell survival, is at the overlap of all three treatments (Fig. S4). This is of potential utility in patient stratification and pharmacogenomics approaches. Omega-3 fatty acids may be a widely deployable preventive treatment, with minimal side-effects, including in women who are or may become pregnant.

\section{New drug discovery/repurposing}

Bioinformatic analyses using the gene expression signature of panels of top biomarkers for low mood/depression (Table 4) identified new potential therapeutics for depression, such as the beta-blocker and serotonin 5HT1A presynaptic receptor antagonist pindolol, the PPAR-alpha activator and lipid lowering agent ciprofibrate, the PPAR- $\gamma$ activator and anti-diabetic pioglitazone, and the anticholinergic and antispasmodic adiphenine. It also identified the natural compounds asiaticoside and chlorogenic acid. 
The last three had also been identified by our previous suicidality studies. Asiaticoside is a triterpenoid component derived from Centella asiatica (Gotu Kola), used in antioxidant, anti-inflammatory, immunomodulatory, and wound healing applications. Chlorogenic acid is an antioxidant polyphenol found in coffee.

\section{Discussion}

We describe a novel and comprehensive effort to discover and validate blood biomarkers of relevance to mood disorders, including testing them in independent cohorts to evaluate predictive ability and clinical utility. These biomarkers also open a window into understanding the biology of mood disorders in general, and of depression and bipolar disorders in particular, as well as indicate new and more precise therapeutic approaches. We provide support for the view that, while mood is a continuum from low to high mood, with some of the best predictive biomarkers for low $\mathrm{mood} / \mathrm{depression}$ and high mood/mania being shared (with changes in opposite direction), some biomarkers are stronger predictors for clinical depression and others for clinical mania, not surprising given the different co-morbidities associated with those conditions.

\section{Current clinical practice and the need for biomarkers}

A convergence of methods assessing the persons' internal subjective feelings and thoughts, along with more objective external ratings of actions and behaviors, is used de facto in clinical practice to assess mood and diagnose clinical mood disorders, such as depression and bipolar disorders. Such an approach is insufficient, and lagging behind those used in other medical specialties. Moreover, $\sim 70 \%$ of patients with bipolar I disorder (BP-I) are initially misdiagnosed, with a mean delay of 5-10 years between illness onset and diagnosis. Most commonly patients are misdiagnosed with major depressive disorder (MDD) [27, 28]. Blood biomarkers related to mood would provide a critical objective measurement to inform clinical assessments and treatment decisions (Fig. 4).

\section{Brain-blood}

Blood biomarkers offer real-world clinical practice advantages. As the brain cannot be readily biopsied in live individuals, and CSF is less easily accessible than blood, we have endeavored over the years to identify blood biomarkers for neuropsychiatric disorders. A whole-blood approach facilitates field deployment of sample collection. The assessment of gene expression changes focuses our approach on immune cells. The ability to identify peripheral gene expression changes that reflect brain activities is likely due to the fact that the brain and immune system have developmental commonalities, marked by shared reactivity and ensuing gene expression patterns. There is also a bidirectional interaction between the brain and immune system. Not all changes in expression in peripheral cells are reflective of or germane to brain activity. By carefully tracking a phenotype with our within-subject design in the discovery step, and then using CFG prioritization, we are able to extract the peripheral changes that do track and are relevant to the brain activity studied, in this case mood state, and its disorders.

Subsequent validation and testing in independent cohorts narrow the list to the best markers. In the end, we do not expect to recapitulate in the blood all that happens in the brain. We just want to have good accessible peripheral biomarkers-“liquid biopsies", as they are called in cancer.

\section{Comprehensive approach}

In this current work, we carried out extensive blood gene expression studies in male and female subjects with major psychiatric disorders, an enriched population in terms of comorbidity with mood disorders and variability of mood. The potential molecular-level co-morbidity between other psychiatric disorders and mood disorders is underlined by the fact that mood medications (antidepressants, mood stabilizers) are used to treat PTSD and schizoaffective disorders, and antipsychotics are used to treat mood disorders. Our goal is first and foremost to discover and validate biomarkers for mood, that are transdiagnostic, in the spirit of RDoC. Second, we aim to understand their universality vs. their specificity by gender and psychiatric diagnosis.

Our studies were stacked in an innovative and comprehensive fashion. First, we endeavored to discover blood gene expression biomarkers for mood using a longitudinal design, looking at differential expression of genes in the blood of male and female subjects with major psychiatric disorders (bipolar disorder, MDD, schizophrenia/schizoaffective, and post-traumatic stress disorder (PTSD)), high risk populations prone to mood disorders, which constitute and enriched pool in which to look for biomarkers. We compared low-mood states to high-mood states using a powerful within-subject design [3-5, 29], to generate a list of differentially expressed genes. Second, we used a comprehensive CFG approach with the whole body of knowledge in the field to date to prioritize from the list of differentially expressed genes/biomarkers of relevance to mood. CFG integrates multiple independent lines of evidence-genetic, gene expression, and protein data, from brain and periphery, from human and animal model studies, as a Bayesian strategy for identifying and prioritizing findings, reducing the false-positives and false-negatives 
inherent in each individual approach. Third, we examined if the expression levels of the top biomarkers identified by us as tracking mood state is changed even more dramatically in blood samples from an independent cohort of subjects with severe depression and with severe mania, to validate these biomarkers. Fourth, the markers thus discovered, prioritized, and validated were tested in corresponding independent cohorts of psychiatric subjects. Fifth, we used the biomarkers to match to existing psychiatric medications, as well as to identify and potentially repurpose new drugs for mood disorders treatment using bioinformatics analyses. The series of studies was a systematic approach to move the field forward toward precision medicine.

\section{Power considerations}

The current work is more comprehensive and powerful in design, and larger in size, than our previous studies [1]. We used a systematic discovery, prioritization, validation, and testing approach, as we have done more recently for suicide and other disorders [3-5, 8-10]. For discovery, we used a hard to accomplish but powerful within-subject design, with an $N$ of 44 subjects with 134 visits. A within-subject design factors out genetic variability, as well as some medications, lifestyle, and demographic effects on gene expression, permitting identification of relevant signal with Ns as small as 1 [29]. Another benefit of a within-subject design may be accuracy/consistency of self-report of psychiatric symptoms ("phene expression"), similar in rationale to the signal detection benefits it provides in gene expression.

Based on our work for the last two decades in genetics and gene expression, along with the results of others in the field, we estimate that the within-subject longitudinal design, by factoring out all genetic and some environmental variability, is up to three orders of magnitude more powerful than an inter-subject case-control cross-sectional design. Moreover, gene expression, by integrating the effects of many SNPs and environment, is up to three orders of magnitude more powerful than a genetic study. Combined, our approach may be up to six orders of magnitude more powerful than a GWAS study, even prior to the CFG literature-based prioritization step. As such, it is at least comparable in power to the largest GWAS to date.

\section{Reproducibility}

Besides our top biomarkers, deeper down in our datasets, we reproduced and expanded our earlier findings of GRK3 (Niculescu et al. Physiological Genomics 2000) [30] and FGFR1 (Le-Niculescu et al. Molecular Psychiatry 2009) [1] as blood biomarkers tracking and predicting mood.

In addition, there is reproducibility with findings generated by independent large scale genetic studies that came out after our analyses were completed, and were thus not included in our CFG approach. A number of their top findings were present in our candidate gene expression biomarkers for mood list that had survived our initial whole-genome, unbiased, within-subject discovery step, before any CFG literature prioritization: 15 out of their 36 top genes for bipolar disorder (Stahl et.al., their Table 1) [31], 187 out of 553 genes for depression (Coleman et.al, their Table S4) [32], 128 out of 268 genes for depression (Howard et al., their Table S9) [33], 487 out of 1291 genes for depression (Chan et al., their Tables S2, S3, S14, S17) [34], 491 out of 819 genes involved in antidepressant response [35], and 79 out of 223 genes for depression (Levey et al. 2020 Medrxiv.org, their Supplementary Tables 1 and 3) (see Supplementary Information- Pathways, Predictions and Reproducibility file). This independent reproducibility of findings between our studies and the genetic studies, which are done in independent cohorts from ours, with independent methodologies, is reassuring, and provides strong convergent evidence for the validity and relevance of our approach and of their genetic approaches. Our work also provides functional evidence for some of their top genetic hits.

\section{Pathophysiological insights}

A number of top candidate biomarkers identified by us have biological roles that are related to the circadian clock (Table S3). To be able to ascertain all the genes in our dataset that were circadian and do estimates for enrichment, we compiled from the literature a database of all the known circadian genes, numbering a total of 1468 genes. Using an estimate of about 21,000 genes in the human genome, that gives about $7 \%$ of genes having some circadian pattern. Out of our 23 top candidate biomarker genes, eight had circadian evidence (35\%), suggesting a fivefold enrichment for circadian genes. This provides a molecular underpinning for the epidemiological data between disrupted sleep and mood disorders, and for the clinical phenomenology of seasonal components to mood disorders.

The majority of top blood biomarkers we have identified have prior evidence in postmortem brain datasets from mood disorders, which indicates their relevance to the pathophysiology of mood disorders (Table S2). The codirectionality of blood changes in our work and brain changes reported in the literature needs to be interpreted with caution, as it may depend on brain region, and on disease stage.

The top biomarkers also had prior evidence of involvement in other psychiatric and related disorders (Table S3), providing a molecular basis for co-morbidity, and the possible precursor effects of some these disorders on mood, and conversely, the precursor role of mood in some of them. In 
particular, a majority of them have an overlap with suicide (92\%), as well as stress (92\%), aging (83\%) and dementia/ Alzheimer (75\%), consistent with them being part of the effects of stress on aging and the "life switch", as described in a previous study by us [26]. The primary medications used to treat stress disorders are serotonin reuptake inhibitors (SSRIs).

\section{Phenomenology (phenomics)}

The mood SMS-7 consists of seven items (Supplementary Fig. S1A). Our clustering analysis revealed the structure of mood symptoms (Supplementary Fig. S1C). Mood and Motivation to do things were the most closely related, followed by Movement activity and Thinking activity. Self-esteem and Interest in pleasurable activities are more distant, and related to each other. Appetite is the most distant, and least related to other items on the scale. Mood reflects and underlies, in essence, if an individual is motivated to get on with life/activities, or not, and if they are happy with themselves. Germane to that, we show that SMS-7 shows good correlation with items of a newly developed visual analog scale for Life Satisfaction (Happiness, Hope, Meaning) (Supplementary Fig. S1D).

\section{Diagnostics}

For the biomarkers identified by us, combining all the available evidence from this current work into a CFE score, brings to the fore biomarkers that have clinical utility for objective assessment and risk prediction for depression, bipolar disorder, and mania (Table 3A-C). These biomarkers should be tested individually as well as tested as polygenic panels of biomarkers in future clinical studies and practical clinical applications in the field. They may permit to distinguish, upon an initial clinical presentation of depression, whether the person is in fact bipolar (Fig. 4). The integration of phenomic data, such as repeated measures of SMS-7 (perhaps via a phone app in a daily fashion), and our CFI-BP score, can further substantiate and elucidate the diagnoses of depression, bipolar disorder, and distinguishing between the two.

In general, our predictive results with biomarkers were stronger in females than in males, by an order of $10-20 \%$ on AUCs. While some of it may be biological, in terms of brain-immune interplay being perhaps higher in women, it is also possible that men are not as accurate as women in terms of self-reporting mood symptoms (affecting our results on state predictions), and do not seek help as much (affecting our results on future hospitalizations predictions). If so, this under-reporting makes the use of objective biomarker tests in men even more necessary.
In regards to how our biomarker discoveries might be applied in clinical laboratory settings, we suggest that panels of top biomarkers, such as BioM12 +1 , be used (Fig. 4). In practice, every new patient tested would be normalized against the database of similar patients already tested, and compared to them for ranking and risk prediction purposes, regardless if a platform like microarrays, RNA sequencing, or a more targeted one like PCR is used in the end clinically. As databases get larger, normative population levels can and should be established, similar to any other laboratory measures. Moreover, longitudinal monitoring of changes in biomarkers within an individual, measuring most recent slope of change, maximum levels attained, and maximum slope of change attained, may be even more informative than simple cross-sectional comparisons of levels within an individual with normative populational levels, as we have shown in our studies. For future point of care approaches, research and development should focus on top individual biomarkers, including at a protein level in accessible fluids such as saliva. One might look at both the best universal biomarkers (that are predictive in all), for reliability, and at the best personalized biomarkers (that are predictive by gender and diagnosis), for higher accuracy.

\section{Treatment}

Biomarkers may also be useful for matching patients to medications and measuring response to treatment (pharmacogenomics) (Fig. 4, Table 3 and S4), as well as new drug discovery and repurposing (Table 4).

\section{Conclusions}

Overall, this work is a major step forward towards understanding, diagnosing, and treating mood disorders. We hope that our trait biomarkers for future risk may be useful in preventive approaches, before the full-blown disorder manifests itself (or re-occurs). Prevention could be accomplished with social, psychological, or biological interventions (i.e., early targeted use of medications or nutraceuticals). Given the fact that 1 in 4 people will have a clinical mood disorder episode in their lifetime, that mood disorders can severely affect quality of life, sometimes leading to suicides, and that not all patients respond to current treatments, the need for and importance of efforts such as ours cannot be overstated.

Acknowledgements We would like to acknowledge our gratitude for the work and results of the many other groups, cited in our paper, who have conducted and published studies (clinical, genetic, and biological) in suicidality. Combining their work with ours makes a convergent approach possible. We would like to thank John Nurnberger and Debomoy Lahiri for useful advice and discussions, as well as Christine 
Daly, Hayley Krushinski, Oliver MacKie, Madelyn Gaines, Hasan Bhullar and Mariah Hill for help with building literature and drug databases. We also would particularly like to thank the subjects in these studies and their families. Without their contribution, such work to advance the understanding of mood disorders would not be possible. This work was supported by NIH grants (1DP2OD007363 and R01MH117431) and a VA Merit Award (2I01CX000139) to ABN.

Author contributions $\mathrm{ABN}$ designed the study and wrote the manuscript. HLN, KR, DFL, SSG, and PLP analyzed the data. JM and PLP assisted with software building and coding. AW, TV, SB, and HD organized, conducted and scored testing in psychiatric subjects. AS assisted with data interpretation. SMK conducted microarray experiments. All authors discussed the results and commented on the manuscript.

\section{Compliance with ethical standards}

Conflict of interest $\mathrm{ABN}$ is listed as inventor on a patent application filed by Indiana University. ABN and AS are co-founders, and SMK is a consultant to, MindX Sciences.

Publisher's note Springer Nature remains neutral with regard to jurisdictional claims in published maps and institutional affiliations.

Open Access This article is licensed under a Creative Commons Attribution 4.0 International License, which permits use, sharing, adaptation, distribution and reproduction in any medium or format, as long as you give appropriate credit to the original author(s) and the source, provide a link to the Creative Commons license, and indicate if changes were made. The images or other third party material in this article are included in the article's Creative Commons license, unless indicated otherwise in a credit line to the material. If material is not included in the article's Creative Commons license and your intended use is not permitted by statutory regulation or exceeds the permitted use, you will need to obtain permission directly from the copyright holder. To view a copy of this license, visit http://creativecommons. org/licenses/by/4.0/.

\section{References}

1. Le-Niculescu H, Kurian SM, Yehyawi N, Dike C, Patel SD, Edenberg HJ, et al. Identifying blood biomarkers for mood disorders using convergent functional genomics. Mol Psychiatry. 2009;14:156-74.

2. Keri S, Szabo C, Kelemen O. Blood biomarkers of depression track clinical changes during cognitive-behavioral therapy. J Affect Disord. 2014;164:118-22.

3. Le-Niculescu H, Levey DF, Ayalew M, Palmer L, Gavrin LM, Jain N, et al. Discovery and validation of blood biomarkers for suicidality. Mol Psychiatry. 2013;18:1249-64.

4. Niculescu AB, Levey DF, Phalen PL, Le-Niculescu H, Dainton $\mathrm{HD}$, Jain N, et al. Understanding and predicting suicidality using a combined genomic and clinical risk assessment approach. Mol Psychiatry. 2015;20:1266-85.

5. Levey DF, Niculescu EM, Le-Niculescu H, Dainton HL, Phalen PL, Ladd TB, et al. Towards understanding and predicting suicidality in women: biomarkers and clinical risk assessment. Mol Psychiatry. 2016;21:768-85.

6. Niculescu AB, Le-Niculescu H, Levey DF, Phalen PL, Dainton HL, Roseberry K, et al. Precision medicine for suicidality: from universality to subtypes and personalization. Mol Psychiatry. 2017;22:1250-73.
7. Niculescu AB, Le-Niculescu H. Convergence of recent GWAS data for suicidality with previous blood biomarkers: independent reproducibility using independent methodologies in independent cohorts. Mol Psychiatry. 2020;25:19-21.

8. Niculescu AB, Le-Niculescu H, Levey DF, Roseberry K, Soe KC, Rogers $\mathrm{J}$, et al. Towards precision medicine for pain: diagnostic biomarkers and repurposed drugs. Mol Psychiatry. 2019;24:501-22.

9. Le-Niculescu H, Roseberry K, Levey DF, Rogers J, Kosary K, Prabha S, et al. Towards precision medicine for stress disorders: diagnostic biomarkers and targeted drugs. Mol Psychiatry. 2019;25:918-38.

10. Niculescu AB, Le-Niculescu H, Roseberry K, Wang S, Hart J, Kaur A, et al. Blood biomarkers for memory: toward early detection of risk for Alzheimer disease, pharmacogenomics, and repurposed drugs. Mol Psychiatry. 2019;25:1651-72.

11. Niculescu AB, Le-Niculescu H. Dissecting suicidality using a combined genomic and clinical approach. Neuropsychopharmacology. 2017;42:360.

12. Niculescu AB, Lulow LL, Ogden CA, Le-Niculescu H, Salomon DR, Schork NJ, et al. PhenoChipping of psychotic disorders: a novel approach for deconstructing and quantitating psychiatric phenotypes. Am J Med Genet Part B, Neuropsychiatr Genet. 2006;141B:653-62.

13. Zhang EE, Liu AC, Hirota T, Miraglia LJ, Welch G, Pongsawakul PY, et al. A genome-wide RNAi screen for modifiers of the circadian clock in human cells. Cell. 2009;139:199-210.

14. McCarthy MJ, Welsh DK. Cellular circadian clocks in mood disorders. J Biol Rhythms. 2012;27:339-52.

15. Le-Niculescu H, McFarland MJ, Ogden CA, Balaraman Y, Patel $\mathrm{S}$, Tan $\mathrm{J}$, et al. Phenomic, convergent functional genomic, and biomarker studies in a stress-reactive genetic animal model of bipolar disorder and co-morbid alcoholism. Am J Med Genet Part B, Neuropsychiatr Genet. 2008;147B:134-66.

16. Belzeaux R, Formisano-Treziny C, Loundou A, Boyer L, Gabert J, Samuelian JC, et al. Clinical variations modulate patterns of gene expression and define blood biomarkers in major depression. J Psychiatr Res. 2010;44:1205-13.

17. Kurian SM, Le-Niculescu H, Patel SD, Bertram D, Davis J, Dike $\mathrm{C}$, et al. Identification of blood biomarkers for psychosis using convergent functional genomics. Mol Psychiatry. 2011;16:37-58.

18. Peters MJ, Joehanes R, Pilling LC, Schurmann C, Conneely KN, Powell $\mathrm{J}$, et al. The transcriptional landscape of age in human peripheral blood. Nat Commun. 2015;6:8570.

19. Niculescu AB. Genomic studies of mood disorders-the brain as a muscle? Genome Biol. 2005;6:215.

20. Beech RD, Lowthert L, Leffert JJ, Mason PN, Taylor MM, Umlauf S, et al. Increased peripheral blood expression of electron transport chain genes in bipolar depression. Bipolar Disord. 2010;12:813-24.

21. Bagot RC, Cates HM, Purushothaman I, Lorsch ZS, Walker DM, Wang J, et al. Circuit-wide transcriptional profiling reveals brain region-specific gene networks regulating depression susceptibility. Neuron. 2016;90:969-83.

22. Labonte B, Engmann O, Purushothaman I, Menard C, Wang J, Tan C, et al. Sex-specific transcriptional signatures in human depression. Nat Med. 2017;23:1102-11.

23. Huttenrauch M, Salinas G, Wirths O. Effects of long-term environmental enrichment on anxiety, memory, hippocampal plasticity and overall brain gene expression in C57BL6 mice. Front Mol Neurosci. 2016;9:62.

24. Erikson GA, Bodian DL, Rueda M, Molparia B, Scott ER, ScottVan Zeeland AA, et al. Whole-genome sequencing of a healthy aging cohort. Cell. 2016;165:1002-11.

25. Nho K, Ramanan VK, Horgusluoglu E, Kim S, Inlow MH, Risacher SL, et al. Comprehensive gene- and pathway-based 
analysis of depressive symptoms in older adults. J Alzheimers Dis. 2015;45:1197-206.

26. Rangaraju S, Levey DF, Nho K, Jain N, Andrews KD, LeNiculescu H, et al. Mood, stress and longevity: convergence on ANK3. Mol Psychiatry. 2016;21:1037-49.

27. Hirschfeld RM, Lewis L, Vornik LA. Perceptions and impact of bipolar disorder: how far have we really come? Results of the national depressive and manic-depressive association 2000 survey of individuals with bipolar disorder. J Clin Psychiatry. 2003;64:161-74.

28. Berk M, Dodd S, Callaly P, Berk L, Fitzgerald P, de Castella AR, et al. History of illness prior to a diagnosis of bipolar disorder or schizoaffective disorder. J Affect Disord. 2007;103:181-6.

29. Chen R, Mias GI, Li-Pook-Than J, Jiang L, Lam HY, Miriami E, et al. Personal omics profiling reveals dynamic molecular and medical phenotypes. Cell. 2012;148:1293-307.

30. Niculescu AB 3rd, Segal DS, Kuczenski R, Barrett T, Hauger RL, Kelsoe JR. Identifying a series of candidate genes for mania and psychosis: a convergent functional genomics approach. Physiol Genom. 2000;4:83-91.

31. Stahl EA, Breen G, Forstner AJ, McQuillin A, Ripke S, Trubetskoy V, et al. Genome-wide association study identifies 30 loci associated with bipolar disorder. Nat Genet. 2019;51:793-803.
32. Coleman JRI, Gaspar HA, Bryois J, Bipolar Disorder Working Group of the Psychiatric Genomics C, Major Depressive Disorder Working Group of the Psychiatric Genomics C, Breen G. The genetics of the mood disorder spectrum: genome-wide association analyses of more than 185,000 cases and 439,000 controls. Biol Psychiatry. 2020;88:169-84.

33. Howard DM, Adams MJ, Clarke TK, Hafferty JD, Gibson J, Shirali M, et al. Genome-wide meta-analysis of depression identifies 102 independent variants and highlights the importance of the prefrontal brain regions. Nat Neurosci. 2019;22:343-52.

34. Chan RF, Turecki G, Shabalin AA, Guintivano J, Zhao M, Xie LY, et al. Cell type-specific methylome-wide association studies implicate neurotrophin and innate immune signaling in major depressive disorder. Biol Psychiatry. 2020;87:431-42.

35. Cook IA, Congdon E, Krantz DE, Hunter AM, Coppola G, Hamilton SP, et al. Time course of changes in peripheral blood gene expression during medication treatment for major depressive disorder. Front Genet. 2019;10:870.

36. Lamb J, Crawford ED, Peck D, Modell JW, Blat IC, Wrobel MJ, et al. The Connectivity Map: using gene-expression signatures to connect small molecules, genes, and disease. Science. 2006;313: $1929-35$.

\section{Affiliations}

H. Le-Niculescu $\mathbb{1}^{1,2} \cdot$ K. Roseberry ${ }^{1} \cdot$ S. S. Gill $\mathbb{1}^{1} \cdot$ D. F. Levey $\mathbb{D}^{1,5} \cdot$ P. L. Phalen $\mathbb{D}^{1,6} \cdot$ J. Mullen ${ }^{1} \cdot$ A. Williams ${ }^{3}$. S. Bhairo ${ }^{1,3} \cdot$ T. Voegtline ${ }^{1,3} \cdot$ H. Davis ${ }^{1,3} \cdot$ A. Shekhar ${ }^{1,7} \cdot$ S. M. Kurian ${ }^{4} \cdot$ A. B. Niculescu ${ }^{1,2,3}$

1 Department of Psychiatry, Indiana University School of Medicine, Indianapolis, IN, USA

2 Stark Neuroscience Research Institute, Indiana University School of Medicine, Indianapolis, IN, USA

3 Indianapolis VA Medical Center, Indianapolis, IN, USA

4 Scripps Health and Department of Molecular Medicine, Scripps Research, La Jolla, CA, USA
5 Present address: Department of Psychiatry, Yale University School of Medicine, New Haven, CT, USA

6 Present address: VA Maryland Health Care System/University of Maryland School of Medicine, Baltimore, MD, USA

7 Present address: Office of the Dean, University of Pittsburgh School of Medicine, Pittsburgh, PA, USA 